Homology, Homotopy and Applications, vol.20(1), 2018, pp.329-358

\title{
A COMPARISON OF TWO MODELS OF ORBISPACES
}

\author{
ALEXANDER KÖRSCHGEN \\ (communicated by Brooke Shipley)
}

\begin{abstract}
This paper proves that the two homotopy theories for orbispaces given by Gepner and Henriques and by Schwede, respectively, agree by providing a zig-zag of Dwyer-Kan equivalences between the respective topologically enriched index categories. The aforementioned authors establish various models for unstable global homotopy theory with compact Lie group isotropy, and orbispaces serve as a common denominator for their particular approaches. Although the two flavors of orbispaces are expected to agree with each other, a concrete comparison zig-zag has not been known so far. We bridge this gap by providing such a zigzag which asserts that all those models for unstable global homotopy theory with compact Lie group isotropy which have been described by the authors named above agree with each other.

On our way, we provide a result which is of independent interest. For a large class of free actions of a compact Lie group, we prove that the homotopy quotient by the group action is weakly equivalent to the strict quotient. This is a known result under more restrictive conditions, e.g., for free actions on a manifold. We broadly extend these results to all free actions of a compact Lie group on a compactly generated Hausdorff space.
\end{abstract}

\section{Contents}

1 Introduction $\quad 330$

1.1 Results . . . . . . . . . . . . . . . . . . . . . 331

1.2 Organization of the paper . . . . . . . . . . . . . 331

$2 \quad$ The intermediate mapping space 332

2.1 A reminder on $\operatorname{Orb}(H, G) \ldots \ldots 332$

2.2 A reminder on $\mathbf{O}_{\mathrm{gl}}(H, G) \ldots \ldots \ldots 335$

2.3 The intermediate mapping space $\operatorname{Orb}^{\prime}(H, G) \ldots \ldots 336$

2.4 The map to $\mathbf{O}_{\mathrm{gl}}(H, G) \ldots \ldots \ldots 338$

The author was supported in part by a grant from the International Max Planck Research School on Moduli Spaces.

Received June 1, 2017, revised October 29, 2017; published on February 28, 2018.

2010 Mathematics Subject Classification: Primary 55R91; Secondary 18D20.

Key words and phrases: orbispace, unstable global homotopy theory, compact Lie group, homotopy quotient.

Article available at http://dx.doi.org/10.4310/HHA.2018.v20.n1.a19

Copyright (C) 2018, Alexander Körschgen. Permission to copy for private use granted. 
3 The zig-zag of Dwyer-Kan equivalences 340

3.1 Compositions on $\mathrm{Orb}^{\prime}$ and on Orb . . . . . . . . . . . . . . . . . . . . . . . . 341

3.2 Compatibility with the composition on $\mathbf{O}_{\mathrm{gl}} \ldots \ldots$. . . . . . . . 345

4 Generalizations of the main results $\quad 346$

$4.1 \mathcal{F}$-relative versions . . . . . . . . . . . . . . 346

4.2 Monomorphism variants . . . . . . . . . . . . . . . . . 347

A Point-set topology $\quad 348$

A.1 Compactly generated weak Hausdorff spaces . . . . . . . . . . . . . . . 349

A.2 Closed inclusions and CGWH colimits . . . . . . . . . . . . . . . 349

A.3 Normal spaces . . . . . . . . . . . . . . . . . . . . . . . 350

A.4 Homotopy quotients of free $G$-spaces . . . . . . . . . . . . . . 351

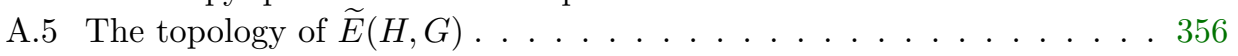

$\begin{array}{ll}\text { References } & 358\end{array}$

\section{Introduction}

Global homotopy theory is concerned with global spaces which can be thought of as spaces that are simultaneously equipped with compatible actions of all compact Lie groups. For any such group $G$, a global space admits a notion of $G$-fixed points, and a map between two global spaces is a global equivalence if it induces a weak equivalence on all of these fixed point spaces. There are also versions for other classes of groups which we elaborate on in Subsection 4.1.

In order to make the notion of global spaces more precise, various models have emerged: In [3], Gepner and Henriques equip stacks and topological groupoids with certain homotopical structures and prove that they yield homotopy theories that are equivalent to their version of orbispaces. This gives rise to three different models for global spaces.

In [9], Schwede introduces $\mathcal{L}$-spaces and orthogonal spaces along with his version of orbispaces, together with model category structures on all of these categories, and verifies that they are Quillen equivalent. Therefore, he also describes three models for global spaces.

In both cases, orbispaces are space-valued enriched presheaves on a topologically enriched small category, the orbit category, equipped with the projective model structure. However, the two variants of orbispaces mentioned above are not equal on the nose because the respective orbit categories differ.

The orbit category used in [3] is called Orb, and the one from [9] is denoted by $\mathbf{O}_{\mathrm{gl}}$. As we will recall, there is significant evidence that the two versions of orbispaces should be equivalent. The objects of both Orb and $\mathbf{O}_{\mathrm{gl}}$ are given by compact Lie groups. Given two such groups $H, G$, the mapping spaces $\mathbf{O}_{\mathrm{gl}}(H, G)$ and $\operatorname{Orb}(H, G)$ have the same weak homotopy type, see Remark 2.11, but, at least to the author's knowledge, no concrete zig-zag of maps has been written down so far.

This exposition shows that there is a zig-zag of weak equivalences between the respective mapping spaces which is compatible with the given structures of topologically enriched categories. We deduce that there is a zig-zag of Dwyer-Kan equivalences 
between the orbit category $\mathbf{O}_{\mathrm{gl}}$ from [9] and the orbit category Orb from [3]. This implies by $[3,5]$ that the associated presheaf categories are Quillen equivalent via a zig-zag, and as a consequence, all the models for global homotopy theory from both papers are equivalent to each other.

\subsection{Results}

For two universal subgroups (see Definition 2.6) $G, H \subseteq \mathbb{L}\left(\mathbb{R}^{\infty}, \mathbb{R}^{\infty}\right)$, set

$$
\mathbf{O}_{\mathrm{gl}}(H, G):=\left(\mathbb{L}\left(\mathbb{R}_{G}^{\infty}, \mathbb{R}_{H}^{\infty}\right) / G\right)^{H},
$$

where $\mathbb{L}$ denotes the space of isometric linear embeddings and $\mathbb{R}_{G}^{\infty}, \mathbb{R}_{H}^{\infty}$ are both just $\mathbb{R}^{\infty}$ equipped with the canonical $G$ - and $H$-action respectively. Moreover, let

$$
\operatorname{Orb}(H, G):=\operatorname{map}(H, G) \times_{G} E G,
$$

where $\operatorname{map}(H, G)$ denotes the space of continuous (and hence smooth) group homomorphisms from $H$ to $G$.

These are the mapping spaces of the topologically enriched categories $\mathbf{O}_{\mathrm{gl}}$ and Orb which share the same set of objects, namely ob $\mathbf{O}_{\mathrm{gl}}=$ ob Orb is the set of all universal subgroups of $\mathbb{L}\left(\mathbb{R}^{\infty}, \mathbb{R}^{\infty}\right)$. Note that a topologically enriched functor which is the identity on objects is a Dwyer-Kan equivalence if and only if it is a weak equivalence on all mapping spaces (see Definition 3.2 for the generic definition of a Dwyer-Kan equivalence).

Theorem. There is a zig-zag of two Dwyer-Kan equivalences, both of which are the identity on objects, between $\mathbf{O}_{\mathrm{gl}}$ and Orb.

This appears as Corollary 3.13.

Corollary. There is a zig-zag of Quillen equivalences between the projective model structures on the enriched presheaf categories $\operatorname{Pre}\left(\mathbf{O}_{\mathrm{gl}}\right.$, Top) and $\operatorname{Pre}(\mathrm{Orb}, \mathrm{Top})$.

This follows immediately using [3, Lemma A.6] or the in-depth account [5, Theorem 3.5]. Here, the first model category, $\operatorname{Pre}\left(\mathbf{O}_{\mathrm{gl}}\right.$, Top) is the variant of orbispaces used in [9] while the second one, Pre(Orb, Top) is used in [3].

We will discuss generalizations of these results for a monomorphism variant of the orbit category and for global family versions in Section 4.

\subsection{Organization of the paper}

The promised zig-zag of Dwyer-Kan equivalences between $\mathbf{O}_{\mathrm{gl}}$ and Orb will consist of a third topologically enriched category Orb' with ob Orb' ${ }^{\prime}=$ ob $\mathbf{O}_{\mathrm{gl}}=\mathrm{ob}$ Orb.

Section 2 focuses on the mapping space $\operatorname{Orb}^{\prime}(H, G)$ for two fixed universal subgroups $H, G \subseteq \mathbb{L}\left(\mathbb{R}^{\infty}, \mathbb{R}^{\infty}\right)$ and its relation to the mapping spaces $\mathbf{O}_{\mathrm{gl}}(H, G)$ and $\operatorname{Orb}(H, G)$. While Subsection 2.1 recalls the definition of $\operatorname{Orb}(H, G)$ along with auxiliary results, Subsection 2.2 proceeds similarly for $\mathbf{O}_{\mathrm{gl}}(H, G)$.

In Subsection 2.3, we construct a free $G$-space $\widetilde{E}(H, G)$, see Definition 2.12, and set $\operatorname{Orb}^{\prime}(H, G):=\widetilde{E}(H, G) \times_{G} E G$. This space admits a canonical map to $\operatorname{Orb}(H, G)$, which is readily seen to be a weak equivalence, see Proposition 2.18. 
Therefore, it remains to specify a weak equivalence $\operatorname{Orb}^{\prime}(H, G) \rightarrow \mathbf{O}_{\text {gl }}(H, G)$, which is the content of Subsection 2.4. We show that the quotient space $\widetilde{E}(H, G) / G$ is canonically homeomorphic to $\mathbf{O}_{\mathrm{gl}}(H, G)$, see Proposition 2.20. After showing that the $G$ action on $\widetilde{E}(H, G)$ is free, we deduce that the natural map $\operatorname{Orb}^{\prime}(H, G)=\widetilde{E}(H, G) \times_{G}$ $E G \rightarrow \widetilde{E}(H, G) / G \cong \mathbf{O}_{\mathrm{gl}}(H, G)$ is a weak equivalence (Proposition 2.22). This concludes the construction of the zig-zag on the level of individual mapping spaces:

$$
\operatorname{Orb}(H, G) \stackrel{\simeq}{E}(H, G) \times_{G} E G=\operatorname{Orb}^{\prime}(H, G) \stackrel{\simeq}{\longrightarrow}(H, G) / G \cong \mathbf{O}_{\mathrm{gl}}(H, G) .
$$

The goal of Section 3 is the extension of this zig-zag to a zig-zag of DwyerKan equivalences. However, the construction of the topologically enriched category $\operatorname{Orb}^{\prime}(H, G)$ is not complete since we have not specified composition laws yet. This is amended in Subsection 3.1: As the composition law on Orb' is similar to the one on Orb, we simultaneously recall the composition on Orb and define the one on Orb'. In the end, we conclude that the maps $\operatorname{Orb}^{\prime}(H, G) \rightarrow \operatorname{Orb}(H, G)$ from Subsection 2.3 extend to a Dwyer-Kan equivalence Orb $\rightarrow$ Orb. Finally, it is left to verify that the composition law on $\mathbf{O}_{\mathrm{gl}}$ is compatible with the maps $\operatorname{Orb}^{\prime}(H, G) \rightarrow \mathbf{O}_{\mathrm{gl}}(H, G)$ introduced in Subsection 2.4, which is the content of Subsection 3.2. In conclusion, we deduce that there is a zig-zag of Dwyer-Kan equivalences:

$$
\mathrm{Orb} \stackrel{\simeq}{\simeq} \mathrm{Orb}^{\prime} \stackrel{\simeq}{\longrightarrow} \mathrm{O}_{\mathrm{gl}}
$$

From the various auxiliary results of the appendix, we would like to highlight a particular one: Theorem A.7 proves that whenever a compact Lie group $G$ acts freely on a Hausdorff space $X$, the canonical map

$$
X \times_{G} E G \rightarrow X / G
$$

is a weak equivalence.

\section{Acknowledgments}

The author would like to acknowledge that the main idea for the construction of the zig-zag of Dwyer-Kan equivalences is due to Thomas Nikolaus. He also provided helpful hints and remarks concerning proof strategies and technical details.

Moreover, the author would like to thank Fabian Hebestreit, Irakli Patchkoria, and Christian Wimmer for fruitful discussions concerning the fiber sequences in Subsection A.4. Finally, the author is grateful for advice and guidance by Stefan Schwede and Peter Teichner.

\section{The intermediate mapping space}

Throughout the main body of this paper, the term space will always refer to a compactly generated weak Hausdorff space. This convention is explained in more detail in Subsection A.1 of the appendix.

\subsection{A reminder on $\operatorname{Orb}(H, G)$}

Definition 2.1. Let $H, G$ be Lie groups. 
(i) Denote by $\operatorname{map}(H, G)$ the space of all continuous (hence smooth) group homomorphisms. It comes with a continuous $G$-action from the right by conjugation, i.e., for $\alpha: H \rightarrow G$ and $g \in G$, define $(\alpha \cdot g)(h):=g^{-1} \alpha(h) g$.

(ii) The quotient of $\operatorname{map}(H, G)$ by this (usually non-free) $G$-action is $\operatorname{Rep}(H, G)$.

(iii) Using the bar construction as a model for $E G$, we set

$$
\operatorname{Orb}(H, G):=\operatorname{map}(H, G) \times_{G} E G:=\left|n \mapsto \operatorname{map}(H, G) \times G^{n}\right|
$$

as the realization of a simplicial topological space.

Remark 2.2. Gepner and Henriques define $\operatorname{Orb}(H, G)$ as the fat geometric realization of the simplicial topological space $\left\{n \mapsto \operatorname{map}(H, G) \times G^{n}\right\}$, which is weakly equivalent to our definition. We prefer the non-fat realization as it behaves better from a technical point of view for our purposes because it commutes with products on the nose. We will elaborate on the consequences in Remark 3.10.

In preparation for later results, we derive an important property of the topology on $\operatorname{map}(H, G)$.

Lemma 2.3 ("Close Maps are Conjugate", [2, Lemma 38.1]). Let $H$ be a compact Lie group and $G$ a Lie group. For each $\alpha \in \operatorname{map}(H, G)$, there is an open neighborhood $U \subseteq H \times G$ of $\operatorname{graph}(\alpha)$ such that for each $\beta \in \operatorname{map}(H, G)$ with $\operatorname{graph}(\beta) \subseteq U, \beta$ is conjugate to $\alpha$.

\section{Proposition 2.4.}

(i) Let $H$ be a compact Lie group and $G$ a Lie group. For each $\alpha \in \operatorname{map}(H, G)$, there is an open neighborhood $V$ of $\alpha$ in $\operatorname{map}(H, G)$ such that each $\beta \in V$ is conjugate to $\alpha$.

(ii) The quotient topology on $\operatorname{Rep}(H, G)=\operatorname{map}(H, G) / G$ is discrete.

(iii) All $G$-orbits $\alpha G$ are open. In particular, there is a $G$-equivariant decomposition

$$
\operatorname{map}(H, G) \cong \coprod_{i \in I} \alpha_{i} G
$$

where $\left\{\alpha_{i}\right\}_{i \in I}$ is a choice of representatives of equivalence classes in $\operatorname{Rep}(H, G)$.

Proof. (i) Pick a metric $d_{G}$ on $G$. As $H$ is compact, the topology on $\operatorname{map}(H, G)$ coincides with the one induced by the metric $d$ which is defined as follows:

$$
d(\alpha, \beta):=\max _{h \in H} d_{G}(\alpha(h), \beta(h)) .
$$

Fix $\alpha \in \operatorname{map}(H, G)$ and choose an open $U$ as in the previous Lemma. For each $h \in H$, pick an open neighborhood $U_{h}=A_{h} \times B_{\varepsilon(h)}(\alpha(h))$ of $(h, \alpha(h))$ inside $U$ where $B_{\varepsilon}(\alpha(h))$ denotes an $\varepsilon$-ball around $\alpha(h)$ with respect to $d_{G}$. By replacing $A_{h}$ with $A_{h} \cap \alpha^{-1}\left(B_{\varepsilon(h) / 2}(\alpha(h))\right)$, we may assume without loss of generality that $\alpha\left(A_{h}\right) \subseteq B_{\varepsilon(h) / 2}(\alpha(h))$.

The $A_{h}$ cover the compact space $H$. Therefore, we find a finite set $H_{0} \subseteq H$ such that $\left\{A_{h} \mid h \in H_{0}\right\}$ covers $H$. Set $\varepsilon:=\min _{h \in H_{0}} \varepsilon(h)$ and denote by

$$
V=B_{\varepsilon / 2}(\alpha)=\left\{\beta \in \operatorname{map}(H, G) \mid d(\alpha, \beta)=\max _{h \in H} d_{G}(\alpha(h), \beta(h))<\varepsilon / 2\right\}
$$

the $\varepsilon / 2$-ball around $\alpha$ in $\operatorname{map}(H, G)$. 
Pick $\beta \in V$. We claim that $\operatorname{graph}(\beta) \subseteq U$. Let $h \in H$, then $h \in A_{h^{\prime}}$ for some $h^{\prime} \in$ $H_{0}$. Also, $d_{G}(\alpha(h), \beta(h))<\varepsilon / 2 \leqslant \varepsilon\left(h^{\prime}\right) / 2$ because $\beta \in V$. As $\alpha\left(A_{h^{\prime}}\right)$ is contained in $B_{\varepsilon\left(h^{\prime}\right) / 2}\left(\alpha\left(h^{\prime}\right)\right)$, we obtain $d_{G}\left(\alpha\left(h^{\prime}\right), \alpha(h)\right)<\varepsilon\left(h^{\prime}\right) / 2$. By the triangle inequality,

$$
d_{G}\left(\alpha\left(h^{\prime}\right), \beta(h)\right) \leqslant d_{G}\left(\alpha\left(h^{\prime}\right), \alpha(h)\right)+d_{G}(\alpha(h), \beta(h))<\varepsilon\left(h^{\prime}\right) / 2+\varepsilon\left(h^{\prime}\right) / 2=\varepsilon\left(h^{\prime}\right) .
$$

Therefore, $(h, \beta(h)) \in A_{h^{\prime}} \times B_{\varepsilon\left(h^{\prime}\right)}\left(\alpha\left(h^{\prime}\right)\right) \subseteq U$, and $\operatorname{graph}(\beta) \subseteq U$. This implies that $\beta$ is conjugate to $\alpha$ by the choice of $U$.

(ii) The quotient projection $\operatorname{map}(H, G) \rightarrow \operatorname{Rep}(H, G)=\operatorname{map}(H, G) / G$ is open. For $\alpha \in \operatorname{map}(H, G)$, an open neighborhood $V$ as in the previous part is sent to the singleton $\{[\alpha]\}$. Hence, all singletons are open in $\operatorname{Rep}(H, G)$.

(iii) As each $[\alpha] \in \operatorname{Rep}(H, G)$ is open, so is its preimage $\alpha G$. The $\alpha G$ are compact, too, as they are the images of the compact sets $\{\alpha\} \times G$ under the action map $\operatorname{map}(H, G) \times G \rightarrow \operatorname{map}(H, G)$. Since $\operatorname{map}(H, G)$ is metrizable, it is Hausdorff, and the compact sets $\alpha G$ must be closed. Choosing $\left\{\alpha_{i}\right\}_{i \in I}$ as in the statement of this proposition, this implies that the canonical map

$$
\coprod_{i \in I} \alpha_{i} G \rightarrow \operatorname{map}(H, G)
$$

is a homeomorphism. The $G$-action on $\operatorname{map}(H, G)$ restricts to $G$-actions on the orbits $\alpha_{i} G$, and the canonical map is $G$-equivariant with respect to these actions.

Of course, the above decomposition always exists on the set level. The new insight is its compatibility with the topology on $\operatorname{map}(H, G)$. This allows us to determine the weak homotopy type of $\operatorname{Orb}(H, G)$ :

Proposition 2.5. Let $\left\{\alpha_{i}\right\}_{i \in I}$ be a choice of representatives of equivalence classes in $\operatorname{Rep}(H, G)$. Then the weak homotopy type of $\operatorname{Orb}(H, G)=\operatorname{map}(H, G) \times_{G} E G$ is

$$
\coprod_{i \in I} B C\left(\alpha_{i}\right)
$$

where $C\left(\alpha_{i}\right) \subseteq G$ denotes the centralizer subgroup of the image of $\alpha_{i}: H \rightarrow G$.

Proof. We follow the argumentation in [7, Remark 2.2.1]: By Proposition 2.4(iii), we have a $G$-equivariant decomposition $\operatorname{map}(H, G) \cong \coprod_{i \in I} \alpha_{i} G$. As the functor $-\times_{G}$ $E G$ commutes with coproducts in the category of $G$-spaces, we obtain

$$
\operatorname{Orb}(H, G)=\operatorname{map}(H, G) \times_{G} E G \cong \coprod_{i \in I}\left(\alpha_{i} G \times_{G} E G\right),
$$

and it is left to verify that $\alpha_{i} G \times_{G} E G$ is a $B C\left(\alpha_{i}\right)$. Note that $\alpha_{i} G \cong C\left(\alpha_{i}\right) \backslash G$ because $C\left(\alpha_{i}\right)$ is the stabilizer of $\alpha_{i}$ with respect to the $G$-action on $\operatorname{map}(H, G)$.

The realization of the simplicial space $E G_{\bullet}$, given by $E G_{n}=G \times G^{n}$, is the space $E G$. It has a free $G$-action which is the realization of the $G$-action on $E G$ • through the first factor of the product. The restriction of this action to $C\left(\alpha_{i}\right) \subseteq G$ is free, so $C\left(\alpha_{i}\right) \backslash E G$ is a $B C\left(\alpha_{i}\right)$.

We claim that $C\left(\alpha_{i}\right) \backslash E G$ is exactly $\alpha_{i} G \times_{G} E G=C\left(\alpha_{i}\right) \backslash G \times_{G} E G$. Restricting the $G$-action on $E G_{\bullet}$ to $C\left(\alpha_{i}\right)$ yields a $C\left(\alpha_{i}\right)$-action on $E G_{\bullet}$. The quotient by this 
action is the simplicial topological space with $n$-th level $C\left(\alpha_{i}\right) \backslash G \times G^{n}$ whose realization is precisely $C\left(\alpha_{i}\right) \backslash G \times{ }_{G} E G$. Taking quotients by $C\left(\alpha_{i}\right)$-actions commutes with realization, so

$$
C\left(\alpha_{i}\right) \backslash G \times_{G} E G=\left|n \mapsto C\left(\alpha_{i}\right) \backslash G \times G^{n}\right|=\left|C\left(\alpha_{i}\right) \backslash E G_{\bullet}\right| \cong C\left(\alpha_{i}\right) \backslash E G .
$$

Therefore, $C\left(\alpha_{i}\right) \backslash G \times_{G} E G$ is a $B C\left(\alpha_{i}\right)$, concluding the proof.

\subsection{A reminder on $\mathrm{O}_{\mathrm{gl}}(H, G)$}

We briefly recall some definitions from [9]:

\section{Definition 2.6.}

(i) For real inner product spaces $V, W$ of finite or countably infinite dimension, $\mathbb{L}(V, W)$ is the space of linear isometric embeddings topologized as in Observation A.15.

In the special case $V=W=\mathbb{R}^{\infty}$, we abbreviate $\mathcal{L}:=\mathbb{L}\left(\mathbb{R}^{\infty}, \mathbb{R}^{\infty}\right)$ and observe that composition endows $\mathcal{L}$ with the structure of a unital topological monoid.

(ii) A compact subgroup $G \subseteq \mathcal{L}$ is a universal subgroup [9, Definition 1.4] if

(a) it admits a necessarily unique structure of a Lie group,

(b) the induced orthogonal $G$-representation on $\mathbb{R}^{\infty}$, denoted by $\mathbb{R}_{G}^{\infty}$, is a complete $G$-universe, i.e., every finite-dimensional $G$-representation embeds into $\mathbb{R}_{G}^{\infty}$ via a $G$-equivariant linear isometry.

(iii) If $H, G$ are such universal subgroups of $\mathcal{L}$, we denote by $E(H, G)=\mathbb{L}\left(\mathbb{R}_{G}^{\infty}, \mathbb{R}_{H}^{\infty}\right)$ the right $H \times G$-space with underlying space $\mathcal{L}=\mathbb{L}\left(\mathbb{R}^{\infty}, \mathbb{R}^{\infty}\right)$ and

$$
(f \cdot(h, g))(x):=h^{-1} \cdot f(g x)
$$

for $f \in \mathbb{L}\left(\mathbb{R}^{\infty}, \mathbb{R}^{\infty}\right),(h, g) \in H \times G$, and $x \in \mathbb{R}^{\infty}$, using the $G$ - and $H$-actions on $\mathbb{R}^{\infty}$.

Finally [9, Definition 2.1],

$$
\mathbf{O}_{\mathrm{gl}}(H, G):=\left(\mathbb{L}\left(\mathbb{R}_{G}^{\infty}, \mathbb{R}_{H}^{\infty}\right) / G\right)^{H}=(E(H, G) / G)^{H} .
$$

Remark 2.7. (i) The concept of universal subgroups of $\mathcal{L}$ from Definition 2.6 encompasses all compact Lie groups in the sense that isomorphism classes of compact Lie groups are in bijection with conjugacy classes of universal subgroups of $\mathcal{L}[\mathbf{9}$, Proposition 1.5].

(ii) An alternative definition of $\mathbf{O}_{\mathrm{gl}}(H, G)$ can be given by the space of $\mathcal{L}$-equivariant maps

$$
\operatorname{map}_{\mathcal{L}}(\mathcal{L} / H, \mathcal{L} / G)
$$

see also [9, Definition 2.1]. The evaluation at $\left[\operatorname{id}_{\mathbb{R} \infty}\right] \in \mathcal{L} / H$ induces a homeomorphism from this space to our definition of $\mathbf{O}_{\mathrm{gl}}(H, G)$.

The crucial property of $E(H, G)$, allowing for an identification of the weak homotopy type of $\mathbf{O}_{\mathrm{gl}}(H, G)$, is its universality with respect to graph subgroups.

Definition 2.8. Let $H, G$ be compact Lie groups. 
(i) The family of graph subgroups ([8, Definition 1.1.25]) is defined to be

$$
\begin{aligned}
\mathcal{F}(H, G):= & \operatorname{graph}(\alpha) \subseteq H \times G \mid \alpha: L \rightarrow G \text { a Lie group } \\
& \text { homomorphism for some closed } L \leqslant H\} .
\end{aligned}
$$

(ii) If $E$ is an $H \times G$-space and $\alpha: L \rightarrow G, L \leqslant H$ closed, then we set $E^{\alpha}:=$ $E^{\operatorname{graph}(\alpha)}$ and, as in Proposition 2.5, we let $C(\alpha) \subseteq G$ denote the centralizer of the image of $\alpha$.

Proposition 2.9 ([8, Proposition 1.1.26(i)] and [9, Proposition A.10]). Given universal subgroups $H, G$ of $\mathcal{L}$, the $H \times G$-space $E(H, G)$ is a universal space for the family $\mathcal{F}(H, G)$, i.e., for $K \leqslant H \times G$,

$$
E(H, G)^{K} \simeq \begin{cases}* & \text { if } K \in \mathcal{F}(H, G), \\ \emptyset & \text { if } K \notin \mathcal{F}(H, G) .\end{cases}
$$

Proposition 2.10 ([8, Proposition 1.5.12(i)]). For any choice of representatives $\left\{\alpha_{i}\right\}_{i \in I}$ of equivalence classes in $\operatorname{Rep}(H, G)=\operatorname{map}(H, G) / G$, the canonical map

$$
\lambda: \coprod_{i \in I} E(H, G)^{\alpha_{i}} / C\left(\alpha_{i}\right) \rightarrow(E(H, G) / G)^{H}
$$

is well-defined and a homeomorphism. Furthermore, $E(H, G)^{\alpha_{i}} / C\left(\alpha_{i}\right)$ is a $B C\left(\alpha_{i}\right)$.

Remark 2.11.

(i) Therefore, the weak homotopy type of $\mathbf{O}_{\mathrm{gl}}(H, G)$ is $\coprod_{i \in I} B C\left(\alpha_{i}\right)$ (see also $[\mathbf{9}$, Remark 2.2]). This agrees with the weak homotopy type of $\operatorname{Orb}(H, G)$ by Proposition 2.5.

(ii) Of course, the map $\lambda$ from before depends on the groups $H, G$ and should be called $\lambda_{H, G}$. However, in order to reduce notational clutter, we chose to only decorate those maps with subscripts that will be considered again for varying groups $H, G$ as we discuss the composition law in the next section (such as the map $\widetilde{p}_{H, G}$ from Definition 2.12). Henceforth, this principle will be applied without further remarks.

\subsection{The intermediate mapping space $\operatorname{Orb}^{\prime}(H, G)$}

In order to compare $\operatorname{Orb}(H, G)$ to $\mathbf{O}_{\mathrm{gl}}(H, G)$, we introduce a fattened up version of $\operatorname{map}(H, G)$ that incorporates $E(H, G)$. Its homotopy quotient will be $\operatorname{Orb}^{\prime}(H, G)$.

Convention. For the remainder of this section, let $H$ and $G$ be universal subgroups of $\mathcal{L}$ unless otherwise stated.

Definition 2.12. The $G$-space $\widetilde{E}(H, G)$ has underlying set

$$
\left\{(\alpha, x) \in \operatorname{map}(H, G) \times E(H, G) \mid x \in E(H, G)^{\alpha}\right\}
$$

topologized as a subspace of $\operatorname{map}(H, G) \times E(H, G)$ and equipped with the diagonal action. It comes with a canonical projection map $\widetilde{p}_{H, G}: \widetilde{E}(H, G) \rightarrow \operatorname{map}(H, G)$.

Remark 2.13. Lemma A.17 will show that this is a CGWH space.

Lemma 2.14. The $G$-action on $\widetilde{E}(H, G)$ is well-defined. 
Proof. Let $\alpha \in \operatorname{map}(H, G)$ and $x \in E(H, G)^{\alpha}$. We need to show that $x g \in E(H, G)^{\alpha g}$. Spelling out the definitions, this amount to verifying that for each $h \in H$, the point $x g=x \cdot(1, g)$ is fixed under the action of $\left(h, g^{-1} \alpha(h) g\right)$. We compute

$$
x \cdot(1, g) \cdot\left(h, g^{-1} \alpha(h) g\right)=x \cdot(h, \alpha(h) g)=x \cdot(h, \alpha(h)) \cdot(1, g)=x \cdot(1, g)=x g .
$$

The $G$-action is automatically continuous because it is the restriction of the continuous diagonal action on $\operatorname{map}(H, G) \times E(H, G)$.

Proposition 2.15. The map $\widetilde{p}_{H, G}$ is a fiber bundle.

Proof. Let us write $\widetilde{p}=\widetilde{p}_{H, G}$ within this proof. By Proposition 2.4, it suffices to show that the restriction

$$
\widetilde{p}^{-1}(\alpha G) \rightarrow \alpha G
$$

is a fiber bundle for any $\alpha \in \operatorname{map}(H, G)$, where $\alpha G \subseteq \operatorname{map}(H, G)$ is the $G$-orbit of $\alpha$.

The canonical map $\operatorname{Stab}(\alpha) \backslash G \rightarrow \alpha G$ is a continuous bijection from a compact space to $\alpha G \subseteq \operatorname{map}(H, G)$. Since the $\operatorname{space} \operatorname{map}(H, G)$ is metrizable, it is Hausdorff and so is its subspace $\alpha G$. Thus, the map is a homeomorphism. Therefore, the map $\tilde{p}^{-1}(\alpha G) \rightarrow \alpha G$ from before is homeomorphic to the projection map

$$
\widetilde{p}_{\alpha}:\left\{(\bar{g}, x) \in(\operatorname{Stab}(\alpha) \backslash G) \times E(H, G) \mid x \in E(H, G)^{\alpha g}\right\} \rightarrow \operatorname{Stab}(\alpha) \backslash G .
$$

Fix a point $\bar{g} \in \operatorname{Stab}(\alpha) \backslash G$. The stabilizer $\operatorname{Stab}(\alpha) \subseteq G$ is a closed subgroup of the Lie group $G$. In particular, the quotient map $\pi: G \rightarrow \operatorname{Stab}(\alpha) \backslash G$ has local sections, and we can choose a neighborhood $U$ of $\bar{g}$ and a section $s: U \rightarrow G$. We are now in the position to write down a local trivialization of $\widetilde{p}_{\alpha}$ :

$$
\begin{array}{ccc}
\widetilde{p}_{\alpha}^{-1}(U)=\left\{(\bar{h}, x) \in U \times E(H, G) \mid x \in E(H, G)^{\alpha h}\right\} & \cong U \times E(H, G)^{\alpha}, \\
(\bar{h}, x) & \mapsto\left(\bar{h}, x \cdot s(\bar{h})^{-1}\right), \\
(\bar{h}, x \cdot s(\bar{h})) & \longleftrightarrow & (\bar{h}, x) .
\end{array}
$$

The maps are continuous because the section $s$ is continuous and it is easy to see that they are mutually inverse. It is left to verify that they are well-defined. For $(\bar{h}, x) \in$ $U \times E(H, G)^{\alpha}$, we have to check that $x \cdot s(\bar{h}) \in E(H, G)^{\alpha h}$. As $s$ is a section, we can write $s(\bar{h})=t h$ for some $t \in \operatorname{Stab}(\alpha)$. Hence, $x \cdot s(\bar{h}) \in E(H, G)^{\alpha t h}=E(H, G)^{\alpha h}$. Well-definedness for the other map works in the same fashion. Finally, it is evident that the homeomorphisms are compatible with the projections to $U$. Therefore, they constitute a local trivialization of $\widetilde{p}_{\alpha}$, concluding the proof.

Corollary 2.16. The map $\widetilde{p}_{H, G}$ is a weak equivalence.

Proof. By the previous proposition, $\widetilde{p}_{H, G}$ is a Serre fibration. Over any point $\alpha \in$ $\operatorname{map}(H, G)$, its fiber is exactly $E(H, G)^{\alpha}$, which is contractible by Proposition 2.9. Consequently, $\widetilde{p}_{H, G}$ is a weak equivalence.

Definition 2.17. Using the bar construction as a model for $E G$, we define

$$
\operatorname{Orb}^{\prime}(H, G):=\widetilde{E}(H, G) \times_{G} E G .
$$


The map $\widetilde{p}_{H, G}: \widetilde{E}(H, G) \rightarrow \operatorname{map}(H, G)$ from Definition 2.12 induces a map

$$
l_{H, G}: \operatorname{Orb}^{\prime}(H, G)=\widetilde{E}(H, G) \times_{G} E G \rightarrow \operatorname{map}(H, G) \times_{G} E G=\operatorname{Orb}(H, G)
$$

by passing to homotopy quotients

Proposition 2.18. The map $l_{H, G}$ is a weak equivalence.

Proof. The weak equivalence $\widetilde{p}_{H, G}: \widetilde{E}(H, G) \rightarrow \operatorname{map}(H, G)$ induces a levelwise weak equivalence

$$
\widetilde{E}(H, G) \times G^{n} \rightarrow \operatorname{map}(H, G) \times G^{n}
$$

between those simplicial topological spaces that realize to the homotopy quotients $\widetilde{E}(H, G) \times_{G} E G$ and $\operatorname{map}(H, G) \times_{G} E G$ respectively.

The unit map $* \rightarrow G$ is a Hurewicz cofibration because $G$ is a smooth manifold. Therefore, any degeneracy map of the two simplicial topological spaces is a Hurewicz cofibration, and both simplicial topological spaces are good. Goodness implies properness, and levelwise weak equivalences between proper simplicial topological spaces realize to weak equivalences. Hence, the induced map $l_{H, G}$ on realizations is a weak equivalence.

\subsection{The map to $\mathrm{O}_{\mathrm{gl}}(H, G)$}

To complete our desired zig-zag of mapping spaces, we still have to construct a map from $\operatorname{Orb}^{\prime}(H, G)$ to $\mathbf{O}_{\mathrm{gl}}(H, G)$. At the end of this subsection, we will have proven that $\widetilde{E}(H, G) / G=\mathbf{O}_{\mathrm{gl}}(H, G)$ so that we can use the canonical map

$$
\operatorname{Orb}^{\prime}(H, G)=\widetilde{E}(H, G) \times_{G} E G \rightarrow \widetilde{E}(H, G) / G=\mathbf{O}_{\mathrm{gl}}(H, G) .
$$

Proposition 2.19. Fix a set $\left\{\alpha_{i}\right\}_{i \in I}$ of representatives of equivalence classes in $\operatorname{map}(H, G) / G=\operatorname{Rep}(H, G)$. Then the canonical map

$$
\mu: \coprod_{i \in I} E(H, G)^{\alpha_{i}} / C\left(\alpha_{i}\right) \rightarrow \widetilde{E}(H, G) / G,
$$

given by its components $\mu_{i}([x])=\left[\alpha_{i}, x\right]$, is a homeomorphism.

Proof. We have to show that the components $\mu_{i}$ are well-defined, implying continuity for $\mu$, and that $\mu$ is bijective with continuous inverse.

For well-definedness, observe that $C\left(\alpha_{i}\right)=\operatorname{Stab}\left(\alpha_{i}\right)$ with respect to the action of $G$ on $\operatorname{map}(H, G)$. Pick $g \in \operatorname{Stab}\left(\alpha_{i}\right)$ and $x \in E(H, G)^{\alpha_{i}}$. Then $\mu_{i}([x g])=\left[\alpha_{i}, x g\right]=$ $\left[\alpha_{i} g, x g\right]=\mu_{i}([x])$. Thus, $\mu_{i}$ is well-defined, and $\mu$ is automatically continuous.

In order to prove surjectivity, pick $[\alpha, x]$ in the codomain of $\mu$. We have $\alpha=\alpha_{i} g$ for some $i \in I$ and $g \in G$. So, $[\alpha, x]=\left[\alpha_{i} g, x\right]=\left[\alpha_{i}, x g^{-1}\right]=\mu_{i}\left(\left[x g^{-1}\right]\right)$ is in the image of $\mu$.

Considering injectivity, suppose that for $i, j \in I$, we have $x \in E(H, G)^{\alpha_{i}}$ and $y \in$ $E(H, G)^{\alpha_{j}}$ such that $\mu_{i}([x])=\mu_{j}([y])$. This means that $\left[\alpha_{i}, x\right]=\left[\alpha_{j}, y\right]$ so there is $g \in G$ such that $\left(\alpha_{i}, x\right)=\left(\alpha_{j} g, y g\right) \in \widetilde{E}(H, G)$. In particular, $\alpha_{i}$ and $\alpha_{j}$ are in the same orbit and we must have $i=j$. Furthermore, $\alpha_{i}=\alpha_{i} g$ implies that $g \in \operatorname{Stab}\left(\alpha_{i}\right)$. As we have $x=y g$, we obtain $[x]=[y] \in E(H, G)^{\alpha_{i}} / \operatorname{Stab}\left(\alpha_{i}\right)=E(H, G)^{\alpha_{i}} / C\left(\alpha_{i}\right)$. 
It remains to show that the inverse map is continuous. To this end, consider the $G$-equivariant decomposition $\operatorname{map}(H, G) \cong \coprod_{i} \alpha_{i} G$, see Proposition 2.4. Each component $\alpha_{i} G$ is canonically homeomorphic to $\operatorname{Stab}\left(\alpha_{i}\right) \backslash G$. Set

$$
\widetilde{E}(H, G)_{i}:=\left\{(\bar{g}, x) \in \operatorname{Stab}\left(\alpha_{i}\right) \backslash G \times E(H, G) \mid x \in E^{\alpha_{i} g}\right\} .
$$

This is a $G$-space via the diagonal action, and we have $\widetilde{E}(H, G) \cong \coprod_{i} \widetilde{E}(H, G)_{i}$ equivariantly. As coproducts commute with quotients, the codomain of $\mu$ is a coproduct of the $\widetilde{E}(H, G)_{i} / G$ with respect to the maps

$$
\begin{array}{ccc}
\iota_{i}: \quad \widetilde{E}(H, G)_{i} / G & \rightarrow & \widetilde{E}(H, G) / G, \\
{[\bar{g}, x]} & \mapsto & {\left[\alpha_{i} g, x\right] .}
\end{array}
$$

A computation reveals that the set-theoretic inverse of $\mu$ has components

$$
\begin{array}{cccc}
\xi_{i}: & \widetilde{E}(H, G)_{i} / G & \rightarrow & E(H, G)^{\alpha_{i}} / \operatorname{Stab}\left(\alpha_{i}\right), \\
{[\bar{g}, x]} & \mapsto & {\left[x g^{-1}\right]}
\end{array}
$$

with respect to the coproduct decomposition of $\widetilde{E}(H, G) / G$ from before. To conclude the proof, we need to show that the $\xi_{i}$ are continuous. This will be achieved by identifying their domains $\widetilde{E}(H, G)_{i} / G$ as certain quotients.

Fix $i \in I$. Since the fixed points $E(H, G)^{\alpha_{i}}$ are closed in $E(H, G)$, the subset $G \times E(H, G)^{\alpha_{i}} \subseteq G \times E(H, G)$ is closed as well. The map $\varrho: G \times E(H, G) \rightarrow G \times$ $E(H, G),(g, x) \mapsto\left(g, x g^{-1}\right)$, is continuous. Hence, $A:=\varrho^{-1}\left(G \times E(H, G)^{\alpha_{i}}\right)$ must be closed. The set $A$ can easily be identified as

$$
A=\left\{(g, x) \in G \times E(H, G) \mid x \in E(H, G)^{\alpha_{i} g}\right\} .
$$

Next, let us consider the quotient map $p$ from $G \times E(H, G)$ to $\left(\operatorname{Stab}\left(\alpha_{i}\right) \backslash G\right) \times$ $E(H, G)$. The subset $A$ of $G \times E(H, G)$ is $p$-saturated, i.e., $p^{-1}(p(A))=A$. To see this, we must show that $(g, x) \in A$ implies $(g h, x h) \in A$ for any $h \in G$. But from $x \in E(H, G)^{\alpha_{i} g}$, it follows that $x h \in E(H, G)^{\alpha_{i} g h}$ and $(g h, x h) \in A$. Now, since $A$ is closed and saturated, the quotient map $p$ restricts to a quotient map $p_{\mid A}: A \rightarrow p(A)$. Moreover, $p(A)=\widetilde{E}(H, G)_{i}$. In the commutative diagram

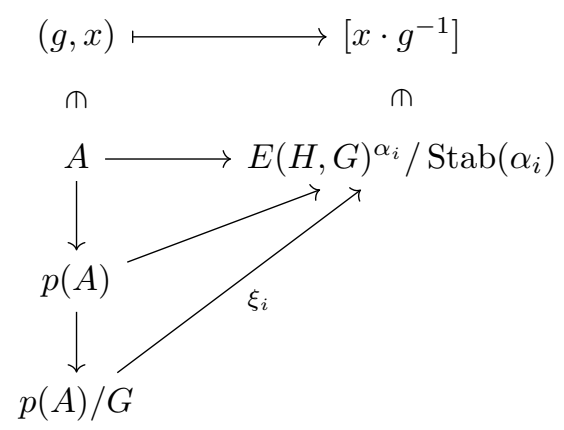

the vertical maps are quotient maps, and the horizontal map is continuous. Therefore, $\xi_{i}$ is continuous, and $\mu$ is a homeomorphism because its set-theoretic inverse is the coproduct of the continuous maps $\xi_{i}$. 
Proposition 2.20. Define $\nu_{H, G}: \widetilde{E}(H, G) / G \rightarrow(E(H, G) / G)^{H}$ by sending $[\alpha, x]$ to $[x]$. Then $\nu_{H, G}$ is a well-defined homeomorphism and fits into a commutative diagram

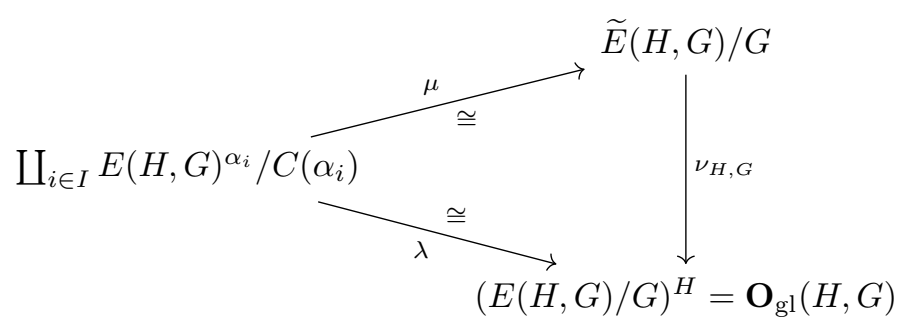

where $\lambda$ is as in Proposition 2.10.

Proof. The map $\mu$ is a homeomorphism by Proposition 2.19, and $\lambda$ is a homeomorphism by Proposition 2.10. Hence, there is a unique homeomorphism $\nu_{H, G}$ making the diagram commute, and one readily checks that the formula is correct.

We have now completed the necessary preparations to define the map that was promised in the beginning of this subsection.

Definition 2.21. The map $k_{H, G}$ is the composition

$$
\operatorname{Orb}^{\prime}(H, G)=\widetilde{E}(H, G) \times_{G} E G \longrightarrow \widetilde{E}(H, G) \times_{G} * \cong \widetilde{E}(H, G) / G \underset{\nu_{H, G}}{\longrightarrow} \mathbf{O}_{\mathrm{gl}}(H, G) .
$$

Proposition 2.22. The map $k_{H, G}$ is a weak equivalence.

Proof. We claim that the $G$-action on $\widetilde{E}(H, G)$ is free. Indeed, if there were $\alpha, g, x$ such that $(\alpha, x) g=(\alpha, x)$, then $x g=x$ would imply that $x \in E(H, G)^{\langle g\rangle}$ where $\langle g\rangle \subseteq G$ denotes the cyclic group generated by $g$. If $g \neq 1$, then $1 \times\langle g\rangle$ cannot be a graph subgroup of $H \times G$. As $E(H, G)$ is a universal space for the family of graph subgroups, $x \in E(H, G)^{\langle g\rangle}=\emptyset$, a contradiction. Hence, $g=1$ and the action is free as desired.

The freeness of the action, Theorem A.7, and Lemma A.17 imply that the comparison map between the homotopy quotient and the point-set quotient is a weak equivalence. Thus, $k_{H, G}$ is a weak equivalence.

For the reader's convenience, we restate the main result of this section: There is a zig-zag of weak equivalences

$$
\operatorname{Orb}(H, G) \stackrel{l_{H, G}}{\simeq} \operatorname{Orb}^{\prime}(H, G) \stackrel{k_{H, G}}{\simeq} \mathbf{O}_{\mathrm{gl}}(H, G) .
$$

The map $l_{H, G}$ and its properties have been established in Subsection 2.3 while $k_{H, G}$ has been discussed in this subsection.

\section{The zig-zag of Dwyer-Kan equivalences}

In this section, we will upgrade our results on mapping spaces to Dwyer-Kan equivalences. In particular, we will interpret the spaces $\operatorname{Orb}(H, G), \operatorname{Orb}^{\prime}(H, G)$, and 
$\mathbf{O}_{\mathrm{gl}}(H, G)$ as mapping spaces of topologically enriched categories and verify that the comparison maps from the previous section give rise to functors sitting in a zig-zag

$$
\mathrm{Orb} \underset{l}{\stackrel{\simeq}{l}} \mathrm{Orb}^{\prime} \stackrel{\simeq}{\underset{k}{\longrightarrow}} \mathrm{O}_{\mathrm{gl}}
$$

of Dwyer-Kan equivalences.

Definition 3.1. The topological categories Orb, Orb', and $\mathbf{O}_{\mathrm{gl}}$ all have the set of objects

$$
\left\{G \subseteq \mathbb{L}\left(\mathbb{R}^{\infty}, \mathbb{R}^{\infty}\right) \mid G \text { a universal subgroup }\right\},
$$

and mapping spaces as defined (or suggested by notation) in the previous section.

It remains to specify composition laws and to verify that identity morphisms exist. We will start by reinterpreting the mapping spaces of Orb' ${ }^{\prime}$ and of Orb as realizations of topological groupoids. This allows for a sleek definition of the respective compositions, and we will also be able to verify compatibility with the maps $l_{H, G}$ easily.

Afterward, we will recall the composition law on $\mathbf{O}_{\mathrm{gl}}$ and check that it is compatible with the maps $k_{H, G}$. This will imply that our comparison zig-zag from the previous section gives rise to a zig-zag of topologically enriched functors. Recall the following

Definition 3.2. Let $\mathcal{C}$ be topologically enriched category. Then the ordinary category $\pi_{0} \mathcal{C}$ has ob $\pi_{0} \mathcal{C}=$ ob $\mathcal{C}$ and $\left(\pi_{0} \mathcal{C}\right)\left(c, c^{\prime}\right)=\pi_{0}\left(\mathcal{C}\left(c, c^{\prime}\right)\right)$ with composition defined in the obvious way.

A functor $f: \mathcal{C} \rightarrow \mathcal{D}$ of topologically enriched categories is a Dwyer-Kan equivalence if the induced functor $\pi_{0} f: \pi_{0} \mathcal{C} \rightarrow \pi_{0} \mathcal{D}$ is an equivalence of categories and $f$ is a weak equivalence on all mapping spaces.

As we have already verified that the maps in our desired zig-zag of functors are weak equivalences on mapping spaces, and since the object functions are identities, we will immediately be able to deduce that the zig-zag of functors is a zig-zag of Dwyer-Kan equivalences.

Convention. For the remainder of this section, $L, K, H, G$ shall be universal subgroups of $\mathbb{L}\left(\mathbb{R}^{\infty}, \mathbb{R}^{\infty}\right)$ unless otherwise specified.

\subsection{Compositions on Orb' and on Orb}

Recall that a topological groupoid $\mathcal{G}$ consists of topological spaces $\mathcal{G}_{0}, \mathcal{G}_{1}$ together with source and target maps $s, t: \mathcal{G}_{1} \rightarrow \mathcal{G}_{0}$ and a composition $\circ: \mathcal{G}_{1}{ }_{s} \times{ }_{t} \mathcal{G}_{1} \rightarrow \mathcal{G}_{0}$, subject to associativity and identity conditions. The category of topological groupoids is denoted by $\operatorname{Grpd}_{\text {Top }}$.

Definition 3.3. Let $G$ be a topological group and $X$ be a right $G$-space. Then the action groupoid $X / / G$ is given by $(X / / G)_{0}=X,(X / / G)_{1}=X \times G$ with $s(x, g)=x$, $t(x, g)=x \cdot g$ and $\left(x^{\prime}, g^{\prime}\right) \circ(x, g)=\left(x, g g^{\prime}\right)$.

Given a $G$-equivariant map $f: K \rightarrow L$ of right $G$-spaces, we define a functor $f / / G: K / / G \rightarrow L / / G$ of topological groupoids by $(f / / G)_{0}=f$ and $(f / / G)_{1}=f \times G$.

Some straightforward computations prove 
Proposition 3.4. The construction from the previous definition gives rise to a functor $-/ / G: G$-Top $\rightarrow \operatorname{Grpd}_{\text {Top }}$. Furthermore, the following diagram commutes

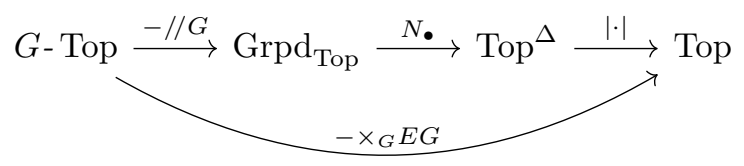

where $N_{\bullet}$ is the topologically enriched version of the nerve functor.

Thus, we see that the morphism spaces of Orb' and of Orb can be described as realizations of certain action groupoids, namely

$$
\operatorname{Orb}(H, G)=\left|N_{\bullet}(\operatorname{map}(H, G) / / G)\right|, \quad \operatorname{Orb}^{\prime}(H, G)=\left|N_{\bullet}(\widetilde{E}(H, G) / / G)\right| .
$$

Definition 3.5. We define two functors of topological groupoids which will yield composition laws:

(i) The functor $\diamond:(\operatorname{map}(H, G) / / G) \times(\operatorname{map}(K, H) / / H) \rightarrow \operatorname{map}(K, G) / / G$ is given by composition in level 0 and by the formula

$$
\begin{array}{cl}
\operatorname{map}(H, G) \times G \times \operatorname{map}(K, H) \times H & \rightarrow \operatorname{map}(K, G) \times G, \\
(\beta, g),(\alpha, h) & \mapsto(\beta \circ \alpha, \beta(h) g)
\end{array}
$$

in level 1.

(ii) The functor $\diamond:(\widetilde{E}(H, G) / / G) \times(\widetilde{E}(K, H) / / H) \rightarrow \widetilde{E}(K, G) / / G$ is defined by

$$
(\beta, y),(\alpha, x) \mapsto(\beta \circ \alpha, x \circ y)
$$

in level 0 and by

$$
(\beta, y, g),(\alpha, x, h) \mapsto(\beta \circ \alpha, x \circ y, \beta(h) g)
$$

in level 1, where $\beta \in \operatorname{map}(H, G), y \in E(H, G)^{\beta}, g \in G, \alpha \in \operatorname{map}(K, H), x \in$ $E(K, H)^{\alpha}$, and $h \in H$. In both formulae, we use the composition of $\mathcal{L}$ which is the underlying space of both $E(H, G)$ and $E(K, H)$.

Remark 3.6. Denote by $\mathbb{B} G=* / / G$ the action groupoid associated to the unique action of $G$ on the one-point space *. Then one can show that $\operatorname{map}(H, G) / / G$ is isomorphic to the internal mapping topological groupoid $\operatorname{map}_{\mathrm{Grpd}_{\mathrm{Top}}}(\mathbb{B} H, \mathbb{B} G)$, and the composition $\diamond$ from part (i) of the previous definition is precisely the composition coming from this enrichment of $\operatorname{Grpd}_{\text {Top }}$ over itself.

Proposition 3.7. The maps from the previous definition are functors.

Proof. We have to verify well-definedness and compatibility with composition and identities for both candidate functors $\diamond$. We will do this for the second one only because the necessary verifications for the first one are part of the verifications for the second one.

In order to check well-definedness in level 0 , we have to prove that for $\beta, y, \alpha, x$ as above, $x \circ y \in E^{\beta \circ \alpha}$. This means that $x \circ y$ is fixed under the action of $\operatorname{graph}(\beta \circ \alpha) \subseteq$ $K \times G$. Pick $k \in K$. We would like to show that $(x \circ y)(k, \beta(\alpha(k)))=(x \circ y)$. Choose 
$v \in \mathbb{R}^{\infty}$. Spelling out the left hand side and evaluating at $v$, we obtain

$$
(x \circ y)\left(k, \beta(\alpha(k))(v)=k^{-1} \cdot(x \circ y)(\beta(\alpha(k)) v)=k^{-1} \cdot(x(y(\beta(\alpha(k)) v))) .\right.
$$

We have $y \in E^{\beta}$ by assumption and $\alpha(k) \in H$. Thus, $(\alpha(k), \beta(\alpha(k))) \in \operatorname{graph}(\beta)$ and $y \cdot(\alpha(k), \beta(\alpha(k)))=y$. Concretely,

$$
\alpha(k)^{-1} \cdot y(\beta(\alpha(k)) v)=y(v),
$$

and, therefore, $y(\beta(\alpha(k)) v)=\alpha(k) y(v)$. Plugging this into the previous computation,

$$
(x \circ y)(k, \beta(\alpha(k)))(v)=k^{-1}(x(\alpha(k) y(v))) .
$$

As $x \in E^{\alpha}$, we have $x \cdot(k, \alpha(k))=x$. Evaluating at $y(v) \in \mathbb{R}^{\infty}$ proves that the right hand side becomes $x(y(v))$, and we have proven that $x \circ y \in E^{\beta \circ \alpha}$.

For the well-definedness in level 1, it is left to verify that the morphism of the topological groupoid $\widetilde{E}(K, G) / / G$ specified by $(\beta \circ \alpha, x \circ y, \beta(h) g)$ has the correct source and the correct target. The source is $(\beta \circ \alpha, x \circ y)=s(\beta, y, g) \diamond s(\alpha, x, h)$ as desired. The target is $((\beta \circ \alpha) \cdot(\beta(h) g),(x \circ y)(\beta(h) g))$ and this should agree with $t(\beta, y, g) \diamond$ $t(\alpha, x, h)=(\beta \cdot g, y \cdot g) \diamond(\alpha \cdot h, x \cdot h)=((\beta \cdot g) \circ(\alpha \cdot h),(x \cdot h) \circ(y \cdot g))$. One readily calculates that $(\beta \circ \alpha) \cdot(\beta(h) g)=(\beta \cdot g) \circ(\alpha \cdot h) \in \operatorname{map}(K, G)$, so the first components agree. For the second components, pick $v \in \mathbb{R}^{\infty}$. We have

$$
((x \cdot h) \circ(y \cdot g))(v)=(x \cdot h)(y(g v))=x(h y(g v)) .
$$

As before, $y \in E^{\beta}$, and $h y(g v)=y(\beta(h) g v)$ consequently. Plugging this in,

$$
((x \cdot h) \circ(y \cdot g))(v)=x(y(\beta(h) g v))=(x \circ y)(\beta(h) g v)=((x \circ y)(\beta(h) g))(v) .
$$

Thus, the candidate functor $\diamond$ is compatible with sources and targets.

Given an object $((\beta, y),(\alpha, x))$ in the product groupoid on the left hand side, its identity is the morphism $((\beta, y, 1),(\alpha, x, 1))$, and applying $\diamond$ yields $(\beta \circ \alpha, x \circ y, 1)$ which is the identity of the object $(\beta \circ \alpha, x \circ y)$.

Pick two composable morphisms in $(\widetilde{E}(H, G) / / G) \times(\widetilde{E}(K, H) / / H)$. Necessarily, they are of the form $((\beta, y, g),(\alpha, x, h))$ and $\left(\left(\beta \cdot g, y \cdot g, g^{\prime}\right),\left(\alpha \cdot h, x \cdot h, h^{\prime}\right)\right)$. Their composition inside the source groupoid is $\left(\left(\beta, y, g g^{\prime}\right),\left(\alpha, x, h h^{\prime}\right)\right)$ which is mapped to $\left(\beta \circ \alpha, x \circ y, \beta\left(h h^{\prime}\right) g g^{\prime}\right)$ by $\diamond$. On the other hand, the composition of the individual images under $\diamond$ inside the target groupoid is

$$
\begin{aligned}
\left((\beta \cdot g) \circ(\alpha \cdot h),(x \cdot h) \circ(y \cdot g),\left((\beta \cdot g)\left(h^{\prime}\right)\right) g^{\prime}\right) \circ & (\beta \circ \alpha, x \circ y, \beta(h) g) \\
& =\left(\beta \circ \alpha, x \circ y, \beta(h) g\left((\beta \cdot g)\left(h^{\prime}\right)\right) g^{\prime}\right) \\
& =\left(\beta \circ \alpha, x \circ y, \beta(h) g g^{-1} \beta\left(h^{\prime}\right) g g^{\prime}\right),
\end{aligned}
$$

which agrees with $\left(\beta \circ \alpha, x \circ y, \beta\left(h h^{\prime}\right) g g^{\prime}\right)$.

Proposition 3.8. The composition laws $\diamond$ defined before give rise to the categories $\mathrm{Orb}_{\mathrm{Grpd}}$ and $\mathrm{Orb}_{\mathrm{Grpd}}^{\prime}$ enriched in topological groupoids with

$$
\mathrm{ob}_{\text {Orb }} \text { Grpd }=\mathrm{ob}_{\text {Orb }}^{\prime}{ }_{\mathrm{Grpd}}=\mathrm{ob} \text { Orb }=\mathrm{ob} \mathrm{Orb}^{\prime},
$$

and

$$
\operatorname{Orb}_{\text {Grpd }}(H, G)=\operatorname{map}(H, G) / / G, \quad \operatorname{Orb}_{\text {Grpd }}^{\prime}(H, G)=\widetilde{E}(H, G) / / G .
$$

Furthermore, the maps $\widetilde{p}_{H, G}: \widetilde{E}(H, G) \rightarrow \operatorname{map}(H, G)$ from Definition 2.12 give rise 
to a functor $p: \operatorname{Orb}_{\mathrm{Grpd}}^{\prime} \rightarrow \mathrm{Orb}_{\mathrm{Grpd}}$ which is the identity on objects and $p_{H, G}:=$ $\widetilde{p}_{H, G} / / G$ on morphism groupoids.

Proof. We have to verify that the composition laws $\diamond$ are associative and unital. Again, we will do this for the second case Orb ${ }_{\text {Grpd }}$ only.

Unitality means that there is a, necessarily unique, functor $* \rightarrow \operatorname{Orb}_{\text {Grpd }}^{\prime}(G, G)$ for the topological groupoid $*$ consisting of one object $*$ and its identity. Specifying such a functor amounts to the selection of an object

$\operatorname{id}_{G} \in \operatorname{Orb}_{\text {Grpd }}^{\prime}(G, G)_{0}=\widetilde{E}(G, G)=\left\{(\alpha, x) \in \operatorname{map}(G, G) \times E(G, G) \mid x \in E(G, G)^{\alpha}\right\}$.

The underlying space of $E(G, G)$ is just $\mathcal{L}=\mathbb{L}\left(\mathbb{R}^{\infty}, \mathbb{R}^{\infty}\right)$, and it is easily verified that id $: \mathbb{R}^{\infty} \rightarrow \mathbb{R}^{\infty} \in \mathcal{L}$ lives in the subspace $E^{\text {id }}$. Thus, we claim that $\mathrm{id}_{G}:=(\mathrm{id}, \mathrm{id})$ yields a unit for $\diamond$.

Let $(\alpha, x) \in \operatorname{Orb}_{\text {Grpd }}^{\prime}(H, G)$. Then $\operatorname{id}_{G} \diamond(\alpha, x)=(\mathrm{id}, \mathrm{id}) \diamond(\alpha, x)=($ id $\circ \alpha, x \circ$ id $)=$ $(\alpha, x)$ as desired. Similarly, $(\alpha, x) \diamond \operatorname{id}_{H}=(\alpha, x)$. On the level of morphisms, the functor $* \rightarrow \operatorname{Orb}_{\text {Grpd }}^{\prime}(G, G)$ selects the identity of $\operatorname{id}_{G}$ which is the morphism (id, id, 1) $\in$ $\operatorname{Orb}_{\text {Grpd }}^{\prime}(G, G)\left(\operatorname{id}_{G}, \operatorname{id}_{G}\right) \subseteq \widetilde{E}(G, G) \times G$. For a morphism $(\alpha, x, h) \in \operatorname{Orb}_{\text {Grpd }}^{\prime}(H, G)$, we compute $(\mathrm{id}, \mathrm{id}, 1) \diamond(\alpha, x, h)=(\mathrm{id} \circ \alpha, x \circ \mathrm{id}, \mathrm{id}(h) 1)=(\alpha, x, h)$ as desired. To verify unitality from the right, we have $(\alpha, x, h) \diamond(\mathrm{id}, \mathrm{id}, 1)=(\alpha, x, \alpha(1) h)=(\alpha, x, h)$ using the identity of $\operatorname{id}_{H}$.

We prove associativity on the level of morphisms only because the statement on objects follows from that. Thus, pick elements $(\alpha, x, k) \in \operatorname{Orb}_{\text {Grpd }}^{\prime}(L, K)_{1},(\beta, y, h) \in$ $\operatorname{Orb}_{\text {Grpd }}^{\prime}(K, H)_{1}$, and $(\gamma, z, g) \in \operatorname{Orb}_{\text {Grpd }}^{\prime}(H, G)_{1}$. We calculate

$$
\begin{aligned}
((\gamma, z, g) \diamond(\beta, y, h)) \diamond(\alpha, x, k) & =(\gamma \circ \beta, y \circ z, \gamma(h) g) \diamond(\alpha, x, k) \\
& =(\gamma \circ \beta \circ \alpha, x \circ y \circ z,(\gamma \circ \beta)(k) \gamma(h) g) \\
& =(\gamma \circ \beta \circ \alpha, x \circ y \circ z, \gamma(\beta(k) h) g) \\
& =(\gamma, z, g) \diamond(\beta \circ \alpha, x \circ y, \beta(k) h) \\
& =(\gamma, z, g) \diamond((\beta, y, h) \diamond(\alpha, x, k))
\end{aligned}
$$

concluding the verification of associativity.

Spelling out the definitions, we see that the map $p_{H, G}=\widetilde{p}_{H, G} / / G$ of topological groupoids is given by

$$
\begin{array}{ccc}
\operatorname{Orb}_{\text {Grpd }}^{\prime}(H, G)_{0} & \rightarrow & \operatorname{Orb}_{\text {Grpd }}(H, G)_{0}, \\
(\alpha, x) & \mapsto & \alpha, \\
\operatorname{Orb}_{\text {Grpd }}^{\prime}(H, G)_{1} & \rightarrow & \operatorname{Orb}_{\text {Grpd }}(H, G)_{1}, \\
(\alpha, x, g) & \mapsto & (\alpha, g),
\end{array}
$$

and it is evident that these formulae are compatible with the composition laws in $\mathrm{Orb}_{\mathrm{Grpd}}$ and $\mathrm{Orb}_{\mathrm{Grpd}}^{\prime}$, respectively, as $H, G$ vary. Therefore, the maps $p_{H, G}$ assemble into a functor $p$ : Orb $_{\text {Grpd }}^{\prime} \rightarrow$ Orb $_{\text {Grpd }}$ as claimed.

Proposition 3.9. Applying the functor $|\cdot| \circ N_{\bullet}$ as in Proposition 3.4 to the composition laws from Definition 3.5 gives rise to categories $\mathrm{Orb}^{\prime}$ and Orb enriched in topological spaces.

Moreover, the functor p from Proposition 3.8 yields a topologically enriched functor $l: \operatorname{Orb}^{\prime} \rightarrow$ Orb whose mapping space components $l_{H, G}: \operatorname{Orb}^{\prime}(H, G) \rightarrow \operatorname{Orb}(H, G)$ are 
exactly the maps $l_{H, G}$ from Definition 2.1\%. In particular, the functor $l$ is a DwyerKan equivalence.

Proof. Both $|\cdot|$ and $N_{\bullet}$ are strongly monoidal. This is straightforward for the functor N. A reference for the monoidality of $|\cdot|$ is [6, Corollary 11.6].

Therefore, applying these functors to the individual mapping spaces of $\mathrm{Orb}_{\mathrm{Grpd}}^{\prime}$ and Orb $_{\text {Grpd }}$ and keeping the object sets the same turns these categories enriched over topological groupoids into categories $\mathrm{Orb}^{\prime}$ and Orb enriched over topological spaces.

We have $\left|N_{\bullet} \operatorname{Orb}_{\text {Grpd }}^{\prime}(H, G)\right|=\left|N_{\bullet}(\widetilde{E}(H, G) / / G)\right|=\widetilde{E}(H, G) \times_{G} E G$ and, similarly, $\left|N_{\bullet} \operatorname{Orb}_{\text {Grpd }}(H, G)\right|=\operatorname{map}(H, G) \times_{G} E G$ by Proposition 3.4. Hence, the mapping spaces $\operatorname{Orb}^{\prime}(H, G)$ and $\operatorname{Orb}(H, G)$ agree with our definitions from Section 2.

Applying $|\cdot| \circ N_{\bullet}$ to $p$ yields a functor $l$ : Orb ${ }^{\prime} \rightarrow$ Orb. As the mapping groupoid components $p_{H, G}: \operatorname{Orb}_{\text {Grpd }}^{\prime}(H, G) \rightarrow \operatorname{Orb}_{\mathrm{Grpd}}(H, G)$ of $p$ come from maps $\widetilde{p}_{H, G}$ of $G$-spaces, we may apply Proposition 3.4 again and deduce that the mapping space components of $l=\left|N_{\bullet}(p)\right|$ are $\left|N_{\bullet}(p)\right|_{H, G}=\left|N_{\bullet}\left(p_{H, G}\right)\right|=\widetilde{p}_{H, G} \times_{G} E G$ which agree with the maps $l_{H, G}$ from Definition 2.17. These are weak equivalences by Proposition 2.18. Consequently, $l$ is a Dwyer-Kan equivalence.

Remark 3.10. In [3, Remark 4.3], Gepner and Henriques choose $\operatorname{Orb}(H, G)$ to be the fat geometric realization $\left\|N_{\bullet}(\operatorname{map}(H, G) / / G)\right\|$. Let us denote this space by $\operatorname{Orb}_{\|\cdot\|}(H, G)$ for the moment. For any good simplicial space $X$, the canonical map $\|X\| \rightarrow|X|$ is a homotopy equivalence, see [10, Proposition A.1(iv)]. In particular, $\operatorname{Orb}_{|\cdot \cdot| \mid}(H, G) \rightarrow \operatorname{Orb}(H, G)$ is a homotopy equivalence.

The composition in $\mathrm{Orb}_{\|\cdot\|}$ depends on a choice of maps $i$ : $\|X \times Y\| \rightarrow\|X\| \times\|Y\|$ and $r:\|X\| \times\|Y\| \rightarrow\|X \times Y\|$ such that $\|X \times Y\|$ is a retract of $\|X\| \times\|Y\|$ via these maps and such that certain associativity conditions hold, see [3, Remarks 2.23 and 4.3]. If one chooses these maps in such a way that the diagram

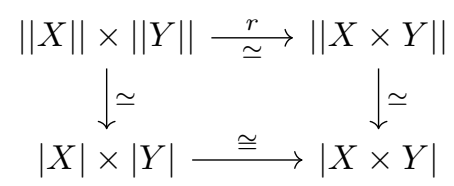

commutes, then one obtains a Dwyer-Kan equivalence Orb $\|\cdot\| \rightarrow$ Orb.

\subsection{Compatibility with the composition on $\mathrm{O}_{\mathrm{gl}}$}

We begin by recalling the composition on $\mathbf{O}_{\mathrm{gl}}$. Having established explicit descriptions of the maps and objects in question, it will be straightforward to verify that the remaining maps $k_{H, G}$ are compatible with identities and composition.

Definition 3.11 ([9, Definition 2.1]). The topologically enriched category $\mathbf{O}_{\mathrm{gl}}$ has objects as in Definition 3.1 and mapping spaces $\mathbf{O}_{\mathrm{gl}}(H, G)=(E(H, G) / G)^{H}$ as in Definition 2.6. Composition is defined as

$$
\begin{array}{ccc}
\mathbf{O}_{\mathrm{gl}}(H, G) \times \mathbf{O}_{\mathrm{gl}}(K, H) & \rightarrow \mathbf{O}_{\mathrm{gl}}(K, G), \\
([y],[x]) & \mapsto & {[x \circ y] .}
\end{array}
$$

The identity morphism in $\mathbf{O}_{\mathrm{gl}}(G, G)$ is given by $\left[\operatorname{id}_{\mathbb{R}^{\infty}}\right]$, the class of the neutral element of the topological monoid $\mathcal{L}=\mathbb{L}\left(\mathbb{R}^{\infty}, \mathbb{R}^{\infty}\right)$, which is the underlying space of $\widetilde{E}(G, G)$. 
Proposition 3.12. The maps $k_{H, G}$ : $\operatorname{Orb}^{\prime}(H, G) \rightarrow \mathbf{O}_{\mathrm{gl}}(H, G)$ from Definition 2.21 give rise to a functor $k: \mathrm{Orb}^{\prime} \rightarrow \mathbf{O}_{\mathrm{gl}}$ that is the identity on objects.

Proof. Recall that $k_{H, G}$ is defined as the composition

$$
\operatorname{Orb}^{\prime}(H, G)=\widetilde{E}(H, G) \times_{G} E G \longrightarrow \widetilde{E}(H, G) \times_{G} * \cong \widetilde{E}(H, G) / G \underset{\nu_{H, G}}{\longrightarrow} \mathbf{O}_{\mathrm{gl}}(H, G)
$$

where $\nu_{H, G}$ is given by $[\alpha, x] \mapsto[x]$, see Proposition 2.20, and the first map is given by $[\alpha, x, u] \mapsto[\alpha, x]$.

We have used the bar construction model $\left|N_{\bullet}(\widetilde{E}(H, G) / / G)\right|$ for $\widetilde{E}(H, G) \times_{G} E G=$ $\operatorname{Orb}^{\prime}(H, G)$, and an arbitrary element of this space is of the form $[\alpha, x, \vec{g}, s]$ for $(\alpha, x) \in \widetilde{E}(H, G), \vec{g} \in G^{n}, s \in \Delta^{n}$. The map $\widetilde{E}(H, G) \times_{G} E G \rightarrow \widetilde{E}(H, G) / G$ becomes $[\alpha, x, \vec{g}, s] \mapsto[\alpha, x]$ under this implicit isomorphism. We conclude that the composite map $k_{H, G}$ has the formula $[\alpha, x, \vec{g}, s] \mapsto[x]$.

In this description, the composition on $\mathrm{Orb}^{\prime}$, which is given by realizing the maps $\diamond$ from Definition 3.5, is given by

$$
\begin{array}{cl}
\operatorname{Orb}^{\prime}(H, G) \times \operatorname{Orb}^{\prime}(K, H) & \rightarrow \operatorname{Orb}^{\prime}(K, H), \\
{[\beta, y, \vec{g}, t],[\alpha, x, \vec{h}, s]} & \mapsto[\beta \circ \alpha, x \circ y, ?],
\end{array}
$$

where the unspecified value ? is irrelevant to our following considerations.

Namely, we need to verify that $k$ is compatible with identities and composition. We leave the straightforward computation for identities to the inclined reader and proceed to the composition. Consider the following diagram:

$$
\begin{aligned}
\operatorname{Orb}^{\prime}(H, G) & \times \operatorname{Orb}^{\prime}(K, H) \stackrel{\circ_{K, H, G}}{\longrightarrow} \operatorname{Orb}^{\prime}(K, G) \\
& \downarrow k_{H, G} \times k_{K, H} \\
\mathbf{O}_{\mathrm{gl}}(H, G) & \times \mathbf{O}_{\mathrm{gl}}(K, H) \stackrel{{ }^{K}, H, G}{\longrightarrow} \mathbf{O}_{\mathrm{gl}}(K, G)
\end{aligned}
$$

Starting with $[\beta, y, \vec{g}, t],[\alpha, x, \vec{h}, s]$ in the upper left corner, we check that it is mapped to $[x \circ y]$ under $k_{K, G} \circ\left(\circ_{K, H, G}\right)$ which is equal to its value under $\circ_{K, H, G} \circ\left(k_{H, G} \times\right.$ $\left.k_{K, H}\right)$. This concludes the proof.

Corollary 3.13. There is a zig-zag of Dwyer-Kan equivalences

$$
\mathrm{Orb} \stackrel{\simeq}{\stackrel{\simeq}{l}} \mathrm{Orb}^{\prime} \stackrel{\underset{k}{\longrightarrow}}{\longrightarrow} \mathrm{O}_{\mathrm{gl}}
$$

as promised at the beginning of this section.

Proof. The functor $l$ exists and is a Dwyer-Kan equivalence by Proposition 3.9. The existence of $k$ was discussed in the previous Proposition. It is a Dwyer-Kan equivalence by Proposition 2.22.

\section{Generalizations of the main results}

\section{1. $\mathcal{F}$-relative versions}

At the beginning of the introduction, we have described global homotopy theory with respect to the class of all compact Lie groups. More generally, one can study global homotopy theory with respect to a chosen global family $\mathcal{F}$, i.e., a non-empty 
class of compact Lie groups which is closed under isomorphisms, subgroups, and quotients, see [9, Remark 3.11]. An $\mathcal{F}$-global space should be thought of as a space that is equipped with simultaneous and compatible actions of all $G \in \mathcal{F}$, and $\mathcal{F}$-global equivalences are defined using $G$-fixed points for $G \in \mathcal{F}$.

Aside from the class $\mathcal{F}_{\text {all }}$ of all compact Lie groups, examples for global families are given by compact abelian Lie groups, finite groups, or trivial groups, respectively.

In our setup, the set of objects of both $\mathbf{O}_{\mathrm{gl}}$ and Orb is given by the set of all universal subgroups $G \subseteq \mathbb{L}\left(\mathbb{R}^{\infty}, \mathbb{R}^{\infty}\right)$. While this set is not isomorphism-closed, it contains a representative for every isomorphism class of compact Lie groups, see Remark 2.7. In particular, we can define versions of $\mathbf{O}_{\mathrm{gl}}$ and Orb relative to a global family and generalize our comparison result: Let $\mathcal{F}$ be a global family, and define $\mathbb{O}_{\mathcal{F}}$ to be the intersection of ob $\mathbf{O}_{\mathrm{gl}}=$ ob Orb with $\mathcal{F}$. Note that for the global family of all compact Lie groups, we have $\mathbb{O}_{\mathcal{F}_{\text {all }}}=$ ob $\mathbf{O}_{\mathrm{gl}}=$ ob Orb.

Corollary 4.1. Let $\mathbb{O}$ be a subset of ob $\mathbf{O}_{\mathrm{gl}}=\mathrm{ob}$ Orb and denote by $\mathbf{O}_{\mathrm{gl}}^{\mathbb{Q}}$ and $\mathrm{Orb}{ }^{\mathbb{Q}}$ the full subcategories of $\mathbf{O}_{\mathrm{gl}}$ and Orb, respectively, on this set of objects.

There is a zig-zag of two Dwyer-Kan equivalences, both of which are the identity on objects, between $\mathbf{O}_{\mathrm{gl}}^{\mathbb{O}}$ and $\mathrm{Orb}^{\mathbb{Q}}$. This zig-zag induces a zig-zag of Quillen equivalences between the projective model structures on the enriched presheaf categories $\operatorname{Pre}\left(\mathbf{O}_{\mathrm{gl}}^{\mathbb{Q}}\right.$, Top) and $\operatorname{Pre}\left(\mathrm{Orb}^{\mathbb{Q}}\right.$, Top $)$. In particular, this is true for $\mathbb{O}=\mathbb{O}_{\mathcal{F}}$ where $\mathcal{F}$ is a global family.

Given a global family $\mathcal{F}$, the category $\mathbf{O}_{\mathrm{gl}}^{\mathbb{O}_{\mathcal{F}}}$ is called $\mathbf{O}_{\mathrm{gl}}^{\mathcal{F}}$ in $[\mathbf{9}$, Remark 3.11]. There, Schwede also equips both the category of $\mathcal{L}$-spaces and the category of orthogonal spaces with model structures relative to $\mathcal{F}$ and establishes that these are equivalent to the projective model structure on $\operatorname{Pre}\left(\mathbf{O}_{\mathrm{gl}}^{\mathcal{F}}\right.$, Top). This yields three different models for $\mathcal{F}$-global homotopy theory.

The setup of Gepner and Henriques is based on the choice of a family of allowed isotropy groups ([3, Subsection 1.3]) that allows for even more general classes of groups which do not necessarily consist of compact Lie groups only. Any global family $\mathcal{F}$ is a family of allowed isotropy groups in their sense, and the category $\operatorname{Pre}\left(\mathrm{Orb}^{\mathbb{Q}_{\mathcal{F}}}, \mathrm{Top}\right)$ is a model for orbispaces with respect to $\mathcal{F}$ in their setup, see [3, Subsection 4.1]. In particular, the cited paper compares this category with two different models for $\mathcal{F}$-global homotopy theory.

\subsection{Monomorphism variants}

Another variant of orbispaces can be obtained by modifying the morphism spaces of the orbit category as discussed in [3, Subsection 2.1]. The morphism space $\operatorname{Orb}(H, G)$ is constructed from the $\operatorname{space} \operatorname{map}(H, G)$ of all morphisms from $H$ to $G$ by taking the homotopy quotient by the $G$-action. Gepner and Henriques define another orbit category by taking the subspace $\operatorname{mono}(H, G) \subseteq \operatorname{map}(H, G)$ of monomorphisms and using $\operatorname{Orb}^{\text {mono }}(H, G):=\operatorname{mono}(H, G) \times_{G} E G$ as the space of maps from $H$ to $G$ in a new index category $\mathrm{Orb}^{\text {mono }}$.

In order to define a monomorphism variant of $\mathbf{O}_{\mathrm{gl}}(H, G)$, consider a decomposition $\operatorname{map}(H, G) \cong \coprod_{i \in I} \alpha_{i} G$ as in Proposition 2.4. Since the $\operatorname{subspace} \operatorname{mono}(H, G) \subseteq$ $\operatorname{map}(H, G)$ is invariant under the $G$-action, there exists a subset $J \subseteq I$ such that 
$\operatorname{mono}(H, G) \cong \coprod_{i \in J} \alpha_{i} G$. By Proposition 2.10, there is a homeomorphism

$$
\lambda: \coprod_{i \in I} E(H, G)^{\alpha_{i}} / C\left(\alpha_{i}\right) \rightarrow(E(H, G) / G)^{H}=\mathbf{O}_{\mathrm{gl}}(H, G),
$$

and we define $\mathbf{O}_{\mathrm{gl}}^{\text {mono }}(H, G)$ to be the image of $\coprod_{i \in J} E(H, G)^{\alpha_{i}} / C\left(\alpha_{i}\right)$ under $\lambda$. This construction was explained to the author by Stefan Schwede.

It remains to find an analogon of $\operatorname{Orb}^{\prime}(H, G)$ for monomorphisms to complete the zig-zag. Similarly to Subsection 2.3, set

$$
\widetilde{E}^{\text {mono }}(H, G):=\left\{(\alpha, x) \in \operatorname{mono}(H, G) \times E(H, G) \mid x \in E(H, G)^{\alpha}\right\},
$$

and $\operatorname{Orb}^{\prime, \text { mono }}(H, G):=\widetilde{E}^{\text {mono }}(H, G) \times_{G} E G$.

By imposing composition laws in analogy to Section 3, we obtain topologically enriched categories $\mathrm{Orb}^{\text {mono }}, \mathbf{O}_{\mathrm{gl}}^{\text {mono }}$, and $\mathrm{Orb}^{\prime, \text { mono }}$ which have the same set of objects as the non-monomorphism versions. The proof of Corollary 3.13 can be adapted without complications to yield

Corollary 4.2. There is a zig-zag

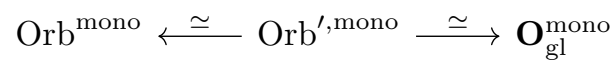

of Dwyer-Kan equivalences, both of which are the identity on objects. This zig-zag induces a zig-zag of Quillen equivalences between the projective model structures on $\operatorname{Pre}\left(\mathrm{Orb}{ }^{\text {mono }}, \mathrm{Top}\right)$ and $\operatorname{Pre}\left(\mathbf{O}_{\mathrm{gl}}^{\text {mono }}, \mathrm{Top}\right)$.

As in the previous subsection, we can restrict to a fixed subset $\mathbb{O}$ of objects in order to account for global families other than $\mathcal{F}_{\text {all }}$ :

Corollary 4.3. Let $\mathbb{O}$ be a subset of ob $\mathbf{O}_{\mathrm{gl}}^{\mathrm{mono}}=\mathrm{ob} \mathrm{Orb}^{\mathrm{mono}}$ and denote by $\mathbf{O}_{\mathrm{gl}}^{\mathbb{Q} \text {,mono }}$ and $\mathrm{Orb}^{\mathbb{Q}, \text { mono }}$ the full subcategories of $\mathbf{O}_{\mathrm{gl}}^{\text {mono }}$ and $\mathrm{Orb}^{\text {mono }}$, respectively, on this set of objects.

There is a zig-zag of two Dwyer-Kan equivalences, both of which are the identity on objects, between $\mathbf{O}_{\mathrm{gl}}^{\mathbb{Q}}$,mono and $\mathrm{Orb}^{\mathbb{O}}$,mono . This zig-zag induces a zig-zag of Quillen equivalences between the projective model structures on the enriched presheaf categories $\operatorname{Pre}\left(\mathbf{O}_{\mathrm{gl}}^{\mathbb{Q} \text {,mono }}\right.$, Top) and $\operatorname{Pre}\left(\mathrm{Orb}^{\mathbb{Q}, \text { mono }}\right.$, Top). In particular, this is true for $\mathbb{O}=\mathbb{O}_{\mathcal{F}}$ where $\mathcal{F}$ is a global family.

\section{Appendix A. Point-set topology}

This appendix is concerned with several technical topics which be deemed to be too distracting for the flow of arguments to be included in the main part of this exposition.

After stating our conventions on the usage of the adjectives compact and compactly generated in Subsection A.1, we proceed by providing helpful statements on the interaction of closed inclusions with colimits in CGWH spaces in Subsection A.2. Afterward, we will recall normal spaces in Subsection A.3 and show that EG is normal as a preparatory result.

Subsection A.4 will show that for a free $G$-space, which is Hausdorff, the comparison map from the homotopy quotient to the point-set quotient is a weak equivalence 
(Theorem A.7). This result is well-known for manifolds or free $G$-CW-complexes, but we could not find a sufficiently general statement in the literature that applies to our circumstances. Therefore, we provide a proof that works for every compactly generated Hausdorff space.

In the remaining Subsection A.5, we will investigate the topology of the spaces $\widetilde{E}(H, G)$ in order to finalize some proofs from the main body of this paper.

\section{A.1. Compactly generated weak Hausdorff spaces}

The main body of this paper takes place in the category of compactly generated weak Hausdorff spaces, also referred to as CGWH spaces. Before we deal with the necessary point-set arguments, let us make the used terminology precise.

A space is compact if every open cover admits a finite subcover. This is also being referred to as quasi-compact in other sources which include the Hausdorff property into the definition of compactness.

Moreover, a space $X$ is compactly generated if, for any subset $Y \subseteq X, Y$ is closed if and only if $u^{-1}(Y)$ is closed for every compact Hausdorff $K$ and every continuous $u: K \rightarrow X$. The space $X$ is weak Hausdorff if for every such $u$ and $K$, the image $u(K)$ is closed in $X$.

These definitions are taken from [11]. Note that this terminology varies within the literature, and some sources refer to compactly generated spaces as $k$-spaces while they take compactly generated spaces to be compactly generated weak Hausdorff spaces in our sense.

Note that the property of being compactly generated is a local property, i.e., a space is compactly generated if and only if each point has a compactly generated neighborhood. The property of being weak Hausdorff is not local, though.

In this paper, we refer to CGWH spaces as (topological) spaces and denote the corresponding category by Top. Within the next subsections, we will have to deal with their point-set subtleties and cite statements about not necessarily CGWH spaces. To this end, we will use the term general topological space for a space that is not necessarily CGWH.

The category of CGWH spaces is cocomplete. Limits and colimits may, however, differ from those computed in the category of general topological spaces. Our convention is that limits and colimits are computed in CGWH unless it is explicitly declared that the diagram in consideration lives in the category of general topological spaces. In this case, limits and colimits are to be taken in the category of general topological spaces. The latter situation does only occur within this Appendix.

For the special case of products, we adopt the following notation from [11]: Given two spaces $X$ and $Y$, we denote by $X \times_{0} Y$ the product taken in the category of general topological spaces, which is not necessarily compactly generated. In contrast, $X \times Y$ shall denote the product in the category of CGWH spaces.

\section{A.2. Closed inclusions and CGWH colimits}

We will now shed some light on situations where specific colimits agree regardless of whether they are computed in CGWH or in the category of general topological spaces. 
Lemma A.1 ([4, Section 2.4, p. 59]). The category of topological spaces is cocomplete. In the case of pushouts along closed inclusions or transfinite compositions of injections, colimits may be computed in the category of general topological spaces since they are already weak Hausdorff.

Lemma A.2. In the category of topological spaces, closed inclusions are closed under pushouts, transfinite compositions, and retracts.

Proof. As weak Hausdorff spaces are automatically $T_{1}$, a closed inclusion in the category of topological spaces is a closed $T_{1}$ inclusion in the category of general topological spaces. Retracts of maps of topological spaces are also retracts of maps of general topological spaces. Also, the relevant pushouts and transfinite compositions can be computed in the category of general topological spaces.

The claim follows from the proof of [4, Lemma 2.4.5] for the cases of pushouts and transfinite compositions and from the proof of [4, Corollary 2.4.6] for the case of retracts.

\section{A.3. Normal spaces}

In this subsection, we will recall the definition of a normal space. Afterward, we will show that normal spaces are abundant and that the class of normal spaces, in contrast to the class of Hausdorff spaces, is closed under certain colimit constructions. As normality implies Hausdorffness for $T_{1}$ spaces, we will use our results to deduce that $E G$ and $\widetilde{E}(H, G)$ are Hausdorff spaces (Lemma A.6 and Lemma A.17 respectively).

Definition A.3. A general topological space $X$ is normal if for any two disjoint closed subsets $A, B \subseteq X$, there are open sets $U, V \subseteq X$ such that $U \cap V=\emptyset, A \subseteq U$, and $B \subseteq V$.

There is a very useful characterization of normal spaces:

Theorem A.4 (Tietze Extension Theorem). $X$ is normal if and only if any continuous map $A \rightarrow \mathbb{R}, A \subseteq X$ closed, can be extended to a map $X \rightarrow \mathbb{R}$.

This theorem easily allows us to deduce a few properties of and recognition criteria for normal spaces:

\section{Lemma A.5.}

(i) Metrizable spaces and compact Hausdorff spaces are normal.

(ii) Closed subspaces of normal spaces are normal.

(iii) If $A \subseteq B$ is a closed inclusion, $B$ and $X$ are normal spaces, then for any pushout diagram

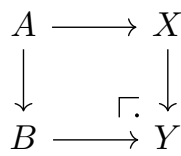

the space $Y$ is normal.

(iv) If $\lambda$ is an ordinal and $\left(X_{\beta}\right)_{\beta<\lambda}$ is a $\lambda$-sequence of normal spaces along closed inclusions, then the space $\operatorname{colim}_{\beta<\lambda} X_{\beta}$ is normal. 
(v) Normal $T_{1}$ spaces are Hausdorff.

(vi) Retracts of normal $T_{1}$ spaces are normal.

(vii) Quillen cofibrant topological spaces are normal.

Proof. (i) is classical, and (ii)-(iv) can easily be shown using the Tietze Extension Theorem.

For example, let us show (iv): Given $\left(X_{\beta}\right)_{\beta<\lambda}$, a closed subspace $A$ of the space $\operatorname{colim}_{\beta<\lambda} X_{\beta}=: X$, and a continuous map $f: A \rightarrow \mathbb{R}$, we wish to find an extension $F: X \rightarrow \mathbb{R}$ of $f$. We start a transfinite recursion in degree 0 . As $A \cap X_{0}$ is closed and $X_{0}$ is normal, we may find an extension $F_{0}: X_{0} \rightarrow \mathbb{R}$ of $f_{\mid A \cap X_{0}}$. Assume that $\beta=\gamma+1$ is a successor ordinal and that there is an extension $F_{\gamma}: X_{\gamma} \rightarrow \mathbb{R}$ of $F_{\mid A \cap X_{\gamma}}$. Then $X_{\gamma} \cup\left(A \cap X_{\beta}\right) \subseteq X_{\beta}$ is closed (because the map $X_{\gamma} \rightarrow X_{\beta}$ has closed image), and the function $F_{\gamma} \cup f_{\mid A \cap X_{\beta}}: X_{\gamma} \cup\left(A \cap X_{\beta}\right) \rightarrow \mathbb{R}$ is continuous (because $X_{\gamma} \rightarrow X_{\beta}$ is a homeomorphism onto its image). Hence, by the normality of $X_{\beta}$, we may extend this function to $F_{\beta}: X_{\beta} \rightarrow \mathbb{R}$. If $\beta$ is a limit ordinal and we have already chosen compatible extensions $F_{\gamma}: X_{\gamma} \rightarrow \mathbb{R}$, then $X_{\beta}=\operatorname{colim}_{\gamma<\beta} X_{\gamma}$ and we extend $f_{A \cap X_{\beta}}$ by $\operatorname{colim}_{\gamma<\beta} F_{\gamma}: X_{\beta} \rightarrow \mathbb{R}$. Finally, taking $F:=\operatorname{colim}_{\beta<\lambda} F_{\lambda}: X \rightarrow \mathbb{R}$ yields the desired extension of $f$.

Part (v) is immediate because points are closed in $T_{1}$ spaces. For part (vi), if $A \stackrel{i}{\rightarrow} X \stackrel{p}{\rightarrow} A$ is a retract diagram, then $i$ is automatically a homeomorphism onto its image. As $X$ is Hausdorff, its retract subspace $i(A)$ is closed. Therefore, it is normal by (ii). The last part follows from (i), (iii), (iv), (vi), and the characterization of cofibrant objects in cofibrantly generated model categories, see [5, Lemma A.4].

Lemma A.6. Let $G$ be a compact Lie group. Then EG is normal and Hausdorff.

Proof. The space $E G$ is the geometric realization of the simplicial topological space $E G$ • which is given by $E G_{n}=G^{n+1}$. As for every geometric realization, $E G$ is the sequential colimit of its skeleta $\operatorname{sk}_{n} E G$, which sit in pushouts

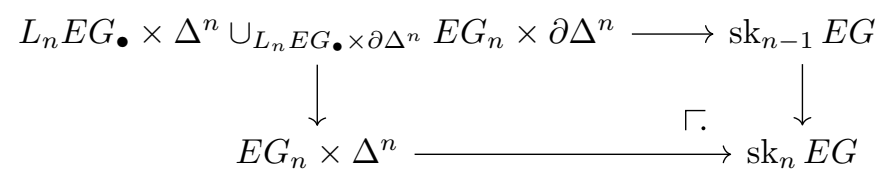

where $L_{n} E G_{\bullet} \rightarrow E G_{n}$ is the $n$-th latching map. Since $E G_{\bullet}$ is proper, cf. the Proof of Proposition 2.18, the latching maps are closed Hurewicz cofibrations. The same is true for the vertical pushout-product morphism on the left hand side. In particular, these pushout-product morphisms are closed inclusions. As $E G_{n} \times \Delta^{n}=G^{n+1} \times \Delta^{n}$ and $\mathrm{sk}_{-1} E G_{\bullet}=\emptyset$ are normal, it follows inductively from Lemma A.5 that the $\mathrm{sk}_{n} E G$ are normal.

Moreover, the pushout diagram tells us that the morphisms $\mathrm{sk}_{n-1} E G \rightarrow \mathrm{sk}_{n} E G$ are closed inclusions, using Lemma A.2. Hence, $E G=\operatorname{colim}_{n} \operatorname{sk}_{n} E G$ is normal by Lemma A.5. As $E G$ is CGWH, it is $T_{1}$. Thus, by Lemma A.5, it is Hausdorff.

\section{A.4. Homotopy quotients of free $G$-spaces}

The goal of this subsection is proving the following theorem: 
Theorem A.7. Let $G$ be a compact Lie group and $X$ a compactly generated Hausdorff space, endowed with a free right $G$-action. Then the canonical map $X \times_{G} E G \rightarrow X / G$ from the homotopy quotient to the quotient is a weak equivalence.

The crucial point in the proof of this theorem is gaining homotopical control over the quotient maps $X \times E G \rightarrow X \times \times_{G} E G$ and $X \rightarrow X / G$. Let us begin by citing several facts about $G$-spaces.

Theorem A.8. Let $G$ be a compact Lie group acting on a general topological space $X$. Denote by $q: X \rightarrow X / G$ the quotient map. Then the following hold:

(i) For any closed subset $A \subset X$, the set $A G=\{a g \mid a \in A, g \in G\}$ is closed in $X$.

(ii) The quotient map $q$ is closed.

(iii) Let $A$ be any $G$-invariant subset of $X$. Then the birestriction $A \rightarrow q(A)$ of $q$ agrees with the quotient map $A \rightarrow A / G$, i.e., the subspace topology on $q(A)$ is the quotient topology of $A / G$.

(iv) If $X$ is a $C G W H$ space, then so is $X / G$. Put differently, the quotient computed in general topological spaces agrees with the quotient taken in $C G W H$ spaces.

(v) If $X$ is Hausdorff, then the quotient map $q$ is proper.

(vi) If $X$ is Hausdorff, then so is $X / G$.

(vii) Assume that the following two conditions are satisfied:

(a) For some fixed $H \leqslant G$, the stabilizer subgroups of all points in $X$ are conjugate to $H$.

(b) The space $X$ is Tychonoff, i.e., completely regular and Hausdorff.

Then $q$ is a fiber bundle with typical fiber $H \backslash G$.

Proof. Statement (i) is a special case of [12, Proposition I.3.1(iii)], and the second assertion (ii) follows from (i) by the definition of the quotient topology. An alternative argument can be found in [8, Proposition B.13(ii)]. Statements (iv) and (iii) are precisely [8, Proposition B.13, (i) and (iii)].

The crucial step in proving (v) is ensuring that the fibers $q^{-1}(q(x))$ are compact. This is rendered possible by the Hausdorff property because it implies that, for any $x \in X$, the canonical map $\operatorname{Stab}(x) \backslash G \rightarrow x G=q^{-1}(q(x))$ is a homeomorphism. A full argument can be found in [12, Section I.3] or [1, Section I.3]. These two sources also provide a reference for (vi). Note that [1] exclusively works in the setting of general topological spaces that are Hausdorff.

Finally, condition (a) is a rephrasing of the requirement that all orbits have type $H \backslash G$, therefore [1, Theorem II.5.8] applies verbatim.

However, the point-set property of being Tychonoff is very inconvenient from a homotopy theorist's point of view because one does not seem to have any grasp on it under limits. Complete regularity means that closed sets can be separated from points by Urysohn functions. Most limits in CGWH spaces necessitate usage of the $k$-ification functor, which potentially introduces more closed sets and, therefore, destroys complete regularity.

On the other hand, compact Hausdorff spaces are automatically Tychonoff, and the topology on a CGWH space is generated by its compact Hausdorff subspaces. This will be the main argument in the proof of the following theorem. 
Theorem A.9. Let $G$ be a compact Lie group acting on a compactly generated Hausdorff space $X$. Assume that for some fixed $H \leqslant G$, all stabilizers subgroups of points in $X$ are conjugate to $H$. Then the quotient map $q: X \rightarrow X / G$ is a fibration.

Proof. The topology on $X$ is generated by its compact subspaces, which are automatically Hausdorff, in the sense that the canonical map

$$
\underset{K \subseteq X \text { compact }}{\text { colim }} K \rightarrow X
$$

is a homeomorphism. For any compact set $K \subseteq X$, there is a $G$-invariant, compact superset $K G$. In particular, the $G$-invariant, compact sets form a cofinal subcategory of the compact sets, and the natural map

$$
\underset{\substack{K \subseteq X \text { compact } \\ \text { and } G \text {-invariant }}}{\text { colim }} K \rightarrow X
$$

is a homeomorphism. This colimit commutes with taking $G$-quotients. Hence,

$$
X / G=\underset{\substack{K \subseteq X \text { compact } \\ \text { and } G \text {-invariant }}}{\text { colim }}(K / G) .
$$

For any $G$-invariant, compact $K \subseteq X$, the space $K$ is compact Hausdorff which implies normal Hausdorff and, consequently, the Tychonoff property. The stabilizer subgroups of the $G$-action on $K$ are conjugate to a fixed $H$, so we may apply Theorem A.8(vii) and deduce that the quotient map $K \rightarrow K / G$ is a fiber bundle and, therefore, a fibration. Moreover, note that this quotient map is exactly the birestriction $q_{\mid K}$ of $q: X \rightarrow X / G$ to $K$ and its image $q(K)=K / G$, see Theorem A.8(iv).

Having made the necessary preparations, let us consider a generating acyclic cofibration of topological spaces $i: A \hookrightarrow B$ and a lifting problem

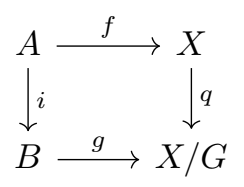

As $B$ is compact, its image $g(B) \subseteq X / G$ is compact. Since $q$ is proper by Theorem A.8(v), the $G$-invariant subset $K:=q^{-1}(g(B))$ is compact, too. Also, $g(B)=$ $q\left(q^{-1}(g(B))\right)=q(K)=K / G$. By corestriction, our lifting problem reduces to the left-hand square of

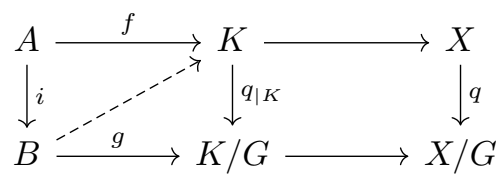

The middle vertical map $q_{\mid K}$ is a fibration by our previous considerations, and we may find a lift as indicated. Postcomposing with the inclusion $K \hookrightarrow X$ immediately solves our original lifting problem.

Remark A.10. In the previous proof, the Hausdorff property for $X$ ensures that the space $K=q^{-1}(g(B))$ is compact Hausdorff. Compactness follows from the properness of $q$, which necessitates the Hausdorff property, and the Hausdorff property for $K$ itself 
is inherited from $X$. Despite several efforts, we were unable to eliminate the Hausdorff requirement for $X$. However, we believe that the statement in its current form may prove useful for other applications as compact Lie group actions on Hausdorff spaces are ubiquitous.

Before we proceed to the proof of Theorem A.7, let us record some preparations which will help us to establish isomorphisms on $\pi_{0}$ and $\pi_{1}$.

Proposition A.11. Let $X$ and $G$ be as before. The $G$-action on $X$ induces a $\pi_{0}(G)$ action on $\pi_{0}(X)$, and there is a dashed arrow making the following triangle commute:

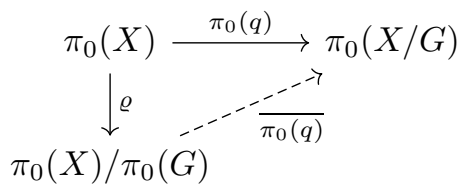

This arrow is an isomorphism and natural with respect to morphisms of G-spaces.

Proof. The $\pi_{0}(G)$-action on $\pi_{0}(X)$ is well-defined because $\pi_{0}$ commutes with products, and $G$-morphisms $X \rightarrow Y$ give rise to morphisms $\pi_{0}(X) \rightarrow \pi_{0}(Y)$ of $\pi_{0}(G)$-sets. If it exists, the arrow $\overline{\pi_{0}(q)}$ is unique which implies naturality.

Denote path-components by [.]. In order to show that $\overline{\pi_{0}(q)}$ exists, pick $[x] \in$ $\pi_{0}(X)$. Then for all $[g] \in \pi_{0}(G)$, we have $\pi_{0}(q)([x])=[q(x)]=[q(x g)]=\pi_{0}(q)([x g])=$ $\pi_{0}(q)([x] \cdot[g])$ where $\cdot$ denotes the $\pi_{0}(G)$-action on $\pi_{0}(X)$. Thus, $\pi_{0}(q)$ factors through the quotient map $\varrho$.

If $X=\emptyset$, then both domain and codomain of $\overline{\pi_{0}(q)}$ are one-point sets and the map is an isomorphism. Otherwise, the quotient map $q: X \rightarrow X / G$ is surjective and induces a surjection on $\pi_{0}$. Consequently, the map $\overline{\pi_{0}(q)}$ is surjective as well, and it remains to show that the map is injective.

Assume that for $x, y \in X$, we have $\overline{\pi_{0}(q)}(\varrho([x]))=\overline{\pi_{0}(q)}(\varrho([y]))$, i.e., $\pi_{0}(q)([x])=$ $\pi_{0}(q)([y])$, which can be rephrased as $[q(x)]=[q(y)]$. This means that there is a path $\gamma:[0 ; 1] \rightarrow X / G$ from $q(x)$ to $q(y)$. As the quotient map $X \rightarrow X / G$ is a fibration, we can lift $\gamma$ to a path $\tilde{\gamma}:[0 ; 1] \rightarrow X$ from $\tilde{\gamma}(0)=x$ to some point $\tilde{\gamma}(1)$ with $q(\tilde{\gamma}(1))=$ $q(y)$. This implies that there is $g \in G$ with $\tilde{\gamma}(1)=y g$. In particular, we have

$$
\varrho([x])=\varrho([\tilde{\gamma}(1)])=\varrho([y g])=\varrho([y] \cdot[g])=\varrho([y]) \in \pi_{0}(X) / \pi_{0}(G),
$$

and we deduce that $\overline{\pi_{0}(q)}$ is injective.

Definition A.12. Let $X$ and $G$ be as before. Assume additionally that $X \neq \emptyset$ and that the $G$-action on $X$ is free. Picking $x \in X$, denote by $\operatorname{fib}_{q(x)}(q)$ the fiber of $q: X \rightarrow X / G$ over $q(x) \in X / G$. We equip this fiber with a group structure using the homeomorphism

$$
G \stackrel{\cong}{\rightarrow} \operatorname{fib}_{q(x)}(q), g \mapsto x g^{-1} .
$$

Remark A.13. The assignment $g \mapsto x g$ is a homeomorphism as well and could have been used to define the group structure. However, the convention described above ensures that the group structure is compatible with the standard conventions for homotopy groups. Moreover, note that this group structure is natural with respect to $G$-equivariant pointed maps of pointed free $G$-spaces. 
Proposition A.14. Let $X, x$, and $G$ be as in Definition A.12. The boundary map

$$
\pi_{1}(X / G, q(x)) \stackrel{\partial}{\rightarrow} \pi_{0}\left(\operatorname{fib}_{q(x)}(q)\right)
$$

associated to the fibration $q: X \rightarrow X / G$ is a group homomorphism with respect to the group structure which is induced by the group structure from Definition A.12 under the functor $\pi_{0}$.

Proof. We interpret $\pi_{1}(X / G, q(x))$ as classes of maps $\left(D^{1}, S^{0}\right) \rightarrow(X / G, q(x))$ of pairs of spaces and employ the notation [.] for classes in $\pi_{0}$ or $\pi_{1}$. Let $\alpha_{1}$ be such a map. As $q$ is a fibration, we may find a lift $\widetilde{\alpha_{1}}: D^{1} \rightarrow X$ such that $q \circ \widetilde{\alpha_{1}}=\alpha_{1}$ and $\widetilde{\alpha_{1}}(0)=x$. Then $\partial\left[\alpha_{1}\right]=\left[\widetilde{\alpha_{1}}(1)\right] \in \operatorname{fib}_{q(x)}(q)$. There is a unique $g \in G$ such that $\widetilde{\alpha_{1}}(1)=x g^{-1}$.

Given another $\alpha_{2}:\left(D^{1}, S^{0}\right) \rightarrow(X / G, q(x))$, choose a lift $\widetilde{\alpha_{2}}$ as before such that $\partial\left[\alpha_{2}\right]=\left[\widetilde{\alpha_{2}}(1)\right]$. Let $h \in G$ be the unique element with $\widetilde{\alpha_{2}}(1)=x h^{-1}$. We wish to show that $\partial\left(\left[\alpha_{1}\right] \cdot\left[\alpha_{2}\right]\right)$ agrees with $\partial\left(\left[\alpha_{1}\right]\right) \cdot \partial\left(\left[\alpha_{2}\right]\right)=\left[x g^{-1}\right] \cdot\left[x h^{-1}\right]$ which is $\left[x(g h)^{-1}\right]=$ $\left[x h^{-1} g^{-1}\right]$ by definition of the group structure on $\pi_{0}\left(\operatorname{fib}_{q(x)}(q)\right)$.

The product $\left[\alpha_{1}\right] \cdot\left[\alpha_{2}\right]$ in $\pi_{1}(X, x)$ is represented by the concatenation $\alpha_{1} * \alpha_{2}$ with the standard convention $\left(\alpha_{1} * \alpha_{2}\right)(0)=\alpha_{1}(0),\left(\alpha_{1} * \alpha_{2}\right)(1)=\alpha_{2}(1)$. Denote by $\widetilde{\alpha_{2}} \cdot g^{-1}$ the path $t \mapsto \widetilde{\alpha_{2}}(t) \cdot g^{-1}$ from $x g^{-1}$ to $x h^{-1} g^{-1}$. Then $\widetilde{\alpha_{1}}$ and $\widetilde{\alpha_{2}} \cdot g^{-1}$ are concatenable and their concatenation $\gamma:=\widetilde{\alpha_{1}} *\left(\widetilde{\alpha_{2}} \cdot g^{-1}\right)$ satisfies $q \circ \gamma=\alpha_{1} * \alpha_{2}$ and $\gamma(0)=x$. Hence, $\partial\left(\left[\alpha_{1}\right] \cdot\left[\alpha_{2}\right]\right)=\partial\left(\left[\alpha_{1} * \alpha_{2}\right)\right]=[\gamma(1)]=\left[x h^{-1} g^{-1}\right]$ as desired.

Proof of Theorem A. \%. In the trivial case $X=\emptyset$, the statement holds. Let us exclude this case from the following considerations.

Observe that $X$ and $E G$ are Hausdorff by assumption and by Lemma A.6, respectively. Therefore, their product $X \times_{0} E G$ in the category of general topological spaces is Hausdorff. As all open sets in $X \times{ }_{0} E G$ are also open in its $k$-ification $X \times E G=$ $k\left(X \times{ }_{0} E G\right)$, we deduce that $X \times E G$ is Hausdorff as well.

Denote the projection $X \times E G \rightarrow X$ by pr and pick $(x, e) \in X \times E G$. We obtain a commutative diagram of fiber sequences:

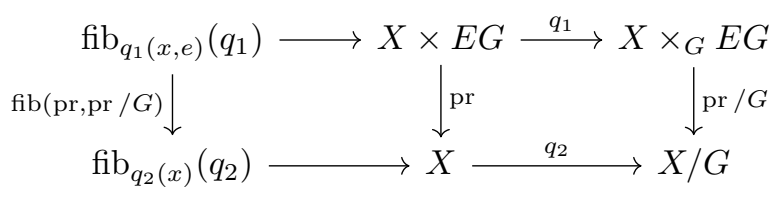

Using homeomorphisms as in Definition A.12, we obtain a commutative triangle

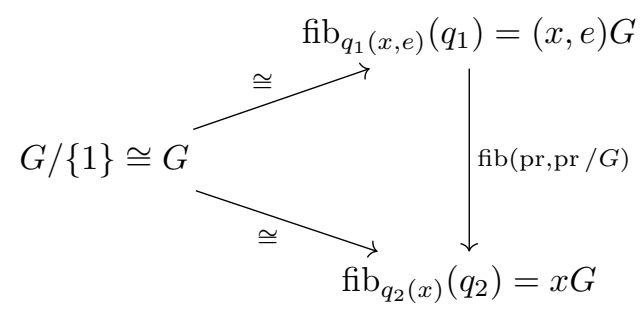

Thus, fib(pr, pr $/ G$ ) is a homeomorphism. Since $E G$ is contractible, pr: $X \times E G \rightarrow X$ is a weak equivalence. By Theorem A.9, the quotient maps $q_{1}$ and $q_{2}$ are fibrations. In the associated long exact sequences on $\pi_{*}$, the terms $\pi_{0}\left(\operatorname{fib}_{q_{1}(x, e)}\left(q_{1}\right)\right)$ and 
$\pi_{0}\left(\operatorname{fib}_{q_{2}(x)}\left(q_{2}\right)\right)$ inherit group structures, and the appropriate boundary maps are group homomorphisms, see Definition A.12 and Proposition A.14. This is sufficient to deduce that $\operatorname{pr} / G$ induces an isomorphism on $\pi_{n}$ for $n \geqslant 1$ and all basepoints by the four lemmas.

Finally, the weak equivalence pr: $X \times E G \rightarrow X$ is $G$-equivariant and induces an isomorphism of $\pi_{0}(G)$-sets on $\pi_{0}$. Thus, it yields an isomorphism $\pi_{0}(\mathrm{pr}) / \pi_{0}(G)$ from $\pi_{0}(X \times E G) / \pi_{0}(G)$ to $\pi_{0}(X) / \pi_{0}(G)$. The diagram

$$
\begin{array}{cc}
\pi_{0}(X \times E G) / \pi_{0}(G) \stackrel{\pi_{0}(\mathrm{pr}) / \pi_{0}(G)}{\cong} \pi_{0}(X) / \pi_{0}(G) \\
\overline{\pi_{0}\left(q_{1}\right)} \mid \simeq & \cong \mid \frac{\downarrow}{\pi_{0}\left(q_{2}\right)} \\
\pi_{0}\left(X \times_{G} E G\right) \stackrel{ }{\pi_{0}(\mathrm{pr} / G)} & \pi_{0}(X / G)
\end{array}
$$

commutes, and the vertical arrows are isomorphisms, see Proposition A.11. Therefore, the map pr $/ G$ is an isomorphism on $\pi_{0}$, too, and it is a weak equivalence.

\section{A.5. The topology of $\widetilde{E}(H, G)$}

In order to apply Theorem A.7 to $\widetilde{E}(H, G)$, which is necessary for the proof of Proposition 2.22, we need to verify that this is a Hausdorff space. Let us start by recalling the topology on $E(H, G)$.

Observation A.15. The underlying space of the $(H \times G)$-space $E(H, G)$ is the space of linear isometries $\mathbb{L}\left(\mathbb{R}^{\infty}, \mathbb{R}^{\infty}\right)$, whose topology we will define now. First, given finitedimensional real inner product spaces $V, W$, the space $\mathbb{L}(V, W)$ of linear isometric embeddings is topologized by choosing an orthonormal basis $\left(v_{1}, \ldots, v_{k}\right)$ of $V$ and using the bijection

$$
\begin{array}{ccc}
\mathbb{L}(V, W) & \rightarrow & \operatorname{Stiefel}_{k}(W), \\
f & \mapsto & \left(f\left(v_{1}\right), \ldots, f\left(v_{k}\right)\right)
\end{array}
$$

to the Stiefel manifold of $k$-frames in $W$. This Stiefel manifold is a subset of $W^{k}$ and endowed with the subspace topology.

Having established the topology on $\mathbb{L}(V, W)$ for $V$ and $W$ finite-dimensional, we move on to the case where the second argument is of countably infinite dimension. For such a $\mathcal{W}$, we define

$$
\mathbb{L}(V, \mathcal{W}):=\operatorname{colim}_{W \in s(\mathcal{W})} \mathbb{L}(V, W),
$$

where $s(\mathcal{W})$ is the poset of finite-dimensional subspaces of $\mathcal{W}$. For $W \leqslant W^{\prime} \in s(\mathcal{W})$, the induced map $\mathbb{L}(V, W) \rightarrow \mathbb{L}\left(V, W^{\prime}\right)$ is a closed inclusion. Furthermore, If $W_{0} \subseteq$ $W_{1} \subseteq \cdots \subseteq \mathcal{W}$ is a strictly increasing sequence of finite-dimensional subspaces such that $\cup_{l} W_{l}=\mathcal{W}$, it is cofinal, and we have

$$
\mathbb{L}(V, \mathcal{W})=\operatorname{colim}_{l} \mathbb{L}\left(V, W_{l}\right) .
$$

As this colimit is taken along closed inclusions, it agrees with the colimit computed in general topological spaces (Lemma A.1). 
It remains to upgrade the first variable to vector spaces of countably infinite dimension. For such a vector space $\mathcal{V}$, we set

$$
\mathbb{L}(\mathcal{V}, \mathcal{W})=\lim _{V \in s(\mathcal{V})} \mathbb{L}(V, \mathcal{W}),
$$

where the limit is taken in the category of CGWH spaces. Again, for an exhaustive sequence $V_{0} \subseteq V_{1} \subseteq \ldots$ of finite dimensional subspaces of $\mathcal{V}$, we have

$$
\mathbb{L}(\mathcal{V}, \mathcal{W})=\lim _{l} \mathbb{L}\left(V_{l}, \mathcal{W}\right) .
$$

By [9, Appendix A], this is the same topology as the subspace topology inherited from the inclusion $\mathbb{L}(\mathcal{V}, \mathcal{W}) \subseteq \operatorname{map}(\mathcal{V}, \mathcal{W})$ into the space of all continuous maps.

Lemma A.16. The space $\mathbb{L}\left(\mathbb{R}^{\infty}, \mathbb{R}^{\infty}\right)$ is Hausdorff.

Proof. First, the spaces $\mathbb{L}\left(\mathbb{R}^{n}, \mathbb{R}^{m}\right)$ are normal as they are metrizable. Therefore, $\mathbb{L}\left(\mathbb{R}^{n}, \mathbb{R}^{\infty}\right)$, which is defined as $\operatorname{colim}_{m} \mathbb{L}\left(\mathbb{R}^{n}, \mathbb{R}^{m}\right)$, is a sequential colimit of normal spaces along closed inclusions. Thus, $\mathbb{L}\left(\mathbb{R}^{n}, \mathbb{R}^{\infty}\right)$ is normal by Lemma A.5. The space $\mathbb{L}\left(\mathbb{R}^{n}, \mathbb{R}^{\infty}\right)$ is $T_{1}$, too, so it is Hausdorff.

The limit $\mathbb{L}\left(\mathbb{R}^{\infty}, \mathbb{R}^{\infty}\right)=\lim _{n} \mathbb{L}\left(\mathbb{R}^{n}, \mathbb{R}^{\infty}\right)$ can be modeled as a (closed) subspace of the infinite product $\prod_{n} \mathbb{L}\left(\mathbb{R}^{n}, \mathbb{R}^{\infty}\right)$ of Hausdorff spaces. As subspaces of Hausdorff spaces are Hausdorff, it suffices to show that $\prod_{n} \mathbb{L}\left(\mathbb{R}^{n}, \mathbb{R}^{\infty}\right)$ is Hausdorff. This is true for the product computed in general topological spaces. The product in the category of CGWH spaces can then be obtained by $k$-ification. However, this procedure does not destroy Hausdorffness because $k$-ification preserves open sets. We conclude that $\prod_{n} \mathbb{L}\left(\mathbb{R}^{n}, \mathbb{R}^{\infty}\right)$ and its subspace $\mathbb{L}\left(\mathbb{R}^{\infty}, \mathbb{R}^{\infty}\right)$ are Hausdorff.

We are now ready to obtain a few important facts about the topology on $\widetilde{E}(H, G)$.

Lemma A.17. The two product topologies $\operatorname{map}(H, G) \times E(H, G)$ and $\operatorname{map}(H, G) \times_{0}$ $E(H, G)$ agree, and they are Hausdorff. The subspace $\widetilde{E}(H, G)$ is compactly generated and Hausdorff. In particular, it is $C G W H$.

Proof. The space $\operatorname{map}(H, G)$ is metrizable and hence locally compact Hausdorff. Therefore, the product $\operatorname{map}(H, G) \times E(H, G)$ agrees with the product $\operatorname{map}(H, G) \times_{0}$ $E(H, G)$ taken in general topological spaces.

For the second statement, first observe that $\operatorname{map}(H, G)$ is Hausdorff by the discussion above and that the space $E(H, G)$ is Hausdorff by Lemma A.16. Therefore, $\operatorname{map}(H, G) \times E(H, G)$ is Hausdorff and so is its subspace $\widetilde{E}(H, G)$. This implies the weak Hausdorff property of course.

In order to prove that $\widetilde{E}(H, G)$ is compactly generated, recall that $\widetilde{E}(H, G)$ is a fiber bundle over $\operatorname{map}(H, G)$ by Proposition 2.15. In particular, the proof shows that each point of $\widetilde{E}(H, G)$ has a neighborhood that is homeomorphic to $U \times E(H, G)^{\alpha}$ for $U \subseteq \operatorname{map}(H, G)$ open and $\alpha \in \operatorname{map}(H, G)$.

According to our convention, the product $U \times E(H, G)^{\alpha}$ is computed in the category of CGWH spaces, and therefore automatically compactly generated. As $U$ is locally compact Hausdorff, we actually have $U \times E(H, G)^{\alpha}=U \times_{0} E(H, G)^{\alpha}$.

In any case, the space $\widetilde{E}(H, G)$ is locally compactly generated which implies that it is compactly generated, concluding the proof. 


\section{References}

[1] G.E. Bredon, Introduction to Compact Transformation Groups, Pure Appl. Math., vol. 46, Academic Press, New York-London, 1972.

[2] P.E. Conner and E.E. Floyd, Differentiable Periodic Maps, Ergeb. Math. Grenzgeb. (N.F.), vol. 33, Springer-Verlag, Berlin-Göttingen-Heidelberg, 1964.

[3] D. Gepner and A. Henriques, Homotopy theory of orbispaces, available at arXiv:math/0701916, 2007.

[4] M. Hovey, Model Categories, reprinted (2007) ed., Math. Surveys Monogr., vol. 63, American Mathematical Society, Providence, RI, 1999.

[5] A. Körschgen, Dwyer-Kan equivalences induce equivalences on topologically enriched presheaves, available at arXiv:1704.07472, 2017.

[6] J.P. May, The Geometry of Iterated Loop Spaces, Lecture Notes in Math., vol. 271, Springer-Verlag, Berlin-Heidelberg-New York, 1972.

[7] C. Rezk, Global homotopy theory and cohesion, available at https://faculty. math.illinois.edu/ rezk/, October 2014.

[8] S. Schwede, Global Homotopy Theory, available at arXiv:1802.09382, 2018

[9] S. Schwede, Orbispaces, orthogonal spaces, and the universal compact Lie group, available at arXiv:1711.06019, 2017

[10] G. Segal, Categories and cohomology theories, Topology 13 (1974), no. 3, 293312 .

[11] N.P. Strickland, The category of CGWH spaces, available at http:// neil-strickland.staff.shef.ac.uk/courses/homotopy/cgwh.pdf, August 2009.

[12] T. tom Dieck, Transformation Groups, de Gruyter Stud. Math., vol. 8, Walter de Gruyter \& Co., Berlin, 1987.

Alexander Körschgen math@koerschgen.name

Mathematisches Institut der Universität Bonn, Endenicher Allee 60, D-53115 Bonn, Germany 